\title{
STRONG STABILITY WITH RESPECT TO WEAK LIMITS FOR A HYPERBOLIC SYSTEM ARISING FROM GAS CHROMATOGRAPHY*
}

\author{
C. BOURDARIAS ${ }^{\dagger}$, M. GISCLON ${ }^{\ddagger}$, AND S. JUNCA ${ }^{\S}$
}

\begin{abstract}
We investigate a system related to a particular isothermal gas-solid chromatography process, called "Pressure Swing Adsorption", with two species and instantaneous exchange kinetics. The particularity of this system is to have a linearly degenerate eigenvalue, which allows the velocity of the gaseous mixture to propagate high frequency waves. In the case of smooth concentrations with a general isotherm, we prove $L^{\infty}$ stability for concentrations, with respect to weak limits of the inlet boundary velocity. Using the Front Tracking Algorithm (FTA), we prove a $L^{1}$ stability for concentrations with bounded variation (BV), under some convex assumptions on the isotherms. In both cases we show that high frequency oscillations with large amplitude of the inlet velocity can propagate without affecting the concentrations.
\end{abstract}

Key words. Systems of conservation laws, boundary conditions, BV estimates, entropy solutions, linearly degenerate fields, convex isotherms, Front Tracking Algorithm, waves interaction, geometric optics.

AMS subject classifications. 35L65, 35L67, 35Q35.

1. Introduction. "Pressure Swing Adsorption (PSA) is a technology used to separate some species from a gas under pressure according to the molecular characteristics and affinity of the species for an adsorbent material. Special adsorbent materials (e.g. zeolites) are used as a molecular sieve, preferentially adsorbing the undesired gases at high pressure. The process then swings to low pressure to desorb the adsorbent material" (source: Wikipedia).

A typical PSA system involves a cyclic process, where a number of connected vessels containing adsorbent material undergo successive pressurization and depressurization steps, in order to produce a continuous stream of purified gas. We focus here on one of the steps of this cyclic process: a step restricted to isothermal behavior. As in general fixed bed chromatography, each of the $d$ species $(d \geq 2)$ simultaneously exists under two phases : a gaseous and movable one with velocity $u(t, x)$ and concentration $c_{i}(t, x)$, and a solid one (adsorbed) with concentration $q_{i}(t, x), 1 \leq i \leq d$. We assume that mass exchanges between the mobile and the stationary phases are infinitely fast, thus the two phases are constantly at composition equilibrium: the concentrations in the solid phase are given by equations as $q_{i}=q_{i}^{*}\left(c_{1}, \ldots, c_{d}\right)$ where the functions $q_{i}^{*}$ are the so-called equilibrium isotherms. Concerning this topic, a theoretical study of a model with finite exchange kinetics was presented in [6] and a numerical approach was developed in [7].

In gas chromatography, velocity variations accompany changes in gas composition, especially in the case of high concentration solute: this is known as the sorption effect. In the present model, the sorption effect is taken into account through a constraint

\footnotetext{
${ }^{*}$ Received February 10, 2009; accepted for publication February 8, 2011.

†Université de Savoie, LAMA, UMR CNRS 5127, 73376 Le Bourget-du-Lac., France (bourdarias@ univ-savoie.fr).

${ }^{\ddagger}$ Université de Savoie, LAMA, UMR CNRS 5127, 73376 Le Bourget-du-Lac., France (gisclon@ univ-savoie.fr).

§IUFM et Université de Nice, Labo. JAD, CNRS UMR 6621, Parc Valrose, 06108 Nice, France (junca@unice.fr).
} 
on the pressure (or on the density in this isothermal case). See [33] and [37] for a precise description of the process and [11] for a survey on various related models.

The system for two species $(d=2)$ with three unknowns $\left(u, c_{1}, c_{2}\right)$ is:

$$
\begin{aligned}
\partial_{t}\left(c_{1}+q_{1}^{*}\left(c_{1}, c_{2}\right)\right)+\partial_{x}\left(u c_{1}\right) & =0, \\
\partial_{t}\left(c_{2}+q_{2}^{*}\left(c_{1}, c_{2}\right)\right)+\partial_{x}\left(u c_{2}\right) & =0, \\
c_{1}+c_{2} & =\rho(t),
\end{aligned}
$$

with suitable initial and boundary data. The function $\rho$ represents the given total density of the mixture. The experimental device is realized such that it is a given function depending only on time and in the sequel we assume that

$$
\rho \equiv 1
$$

(which is not really restrictive from a theoretical point of view).

Notice that we seek positive solutions $\left(c_{1}, c_{2}\right)$, thus considering $(3)$ and $(4), c_{1}, c_{2}$ must satisfy

$$
0 \leq c_{1} \leq 1, \quad 0 \leq c_{2} \leq 1
$$

Let us rewrite system (1), (2), (3) as a $2 \times 2$ system as in [9, 11]. For this purpose, we use the following notations, introduced in [10]: we set $c=c_{1} \in[0,1]$, then $c_{2}=$ $\rho-c_{1}=1-c$, and we set

$$
\begin{aligned}
q_{i}(c) & =q_{i}^{*}(c, 1-c), \quad i=1,2, \\
h(c) & =q_{1}(c)+q_{2}(c) \\
I(c) & =c+q_{1}(c) .
\end{aligned}
$$

Adding (1) and (2), thanks to (3), we get:

$$
\partial_{t}\left(q_{1}(c)+q_{2}(c)\right)+\partial_{x} u=0,
$$

thus our purpose is to study the following system:

$$
\left\{\begin{aligned}
\partial_{t} I(c)+\partial_{x}(u c) & =0 \\
\partial_{t} h(c)+\partial_{x} u & =0
\end{aligned}\right.
$$

supplemented by initial and boundary data:

$$
\left\{\begin{array}{l}
c(0, x)=c_{0}(x) \in[0,1], \quad x>0, \\
c(t, 0)=c_{b}(t) \in[0,1], \quad t>0, \\
u(t, 0)=u_{b}(t)>0, \quad t>0 .
\end{array}\right.
$$

Notice that in (7) we assume an incoming flux at the boundary, i.e. $\forall t>0, u_{b}(t)>0$. In the case where the first species is inert, that is $q_{1}=0$, the $I$ function reduces to the identity function.

The first results of solutions' existence, with large initial and boundary data, which satisfy some entropy criterium in the case of two chemical species, were obtained in $[9,10]$ for System (6) with initial and boundary $B V$ data for the concentration and only $L^{\infty}$ boundary data for the velocity $u_{b}$. Furthermore, the velocity $u$ becomes $B V$ 
with respect to $x$ in the quarter $t>0$ and $x>0$, see $[9,10]$. But, we have no gain of regularity with respect to time for the velocity. The following simple example is illuminating the topic. Let $\underline{c}$ be a constant and $u_{b}$ any $L^{\infty}$ positive function, then

$$
(c(t, x), u(t, x))=\left(\underline{c}, u_{b}(t)\right)
$$

is a weak entropy solution of (6). Thus, strong singularities for the velocity $u$, with respect to time, are expected, singularities which do not seem to affect the concentration.

In this paper, we study more precisely the specific structure of the velocity in two cases, namely the case with smooth concentrations and the more complicated case with $B V$ concentrations. The specific structure of the velocity has an interesting application: the stability in strong topologies of concentrations with respect to weak ${ }^{*}$ limits for the incoming velocity. For instance, physically, a high oscillating velocity can be replaced by its mean value to compute the concentration. Notice that in the chromatography field, there are models with constant velocity $([37,34,11])$.

In the first case (smooth concentration), if the velocity $u(.,$.$) is also smooth, we$ have already proven in [9] that the System (6) reduces to a nonlinear scalar conservation law for the concentration. Actually, for a weak entropy solution, with lipschitz concentration but only $L^{\infty}$ velocity, the reduction to one equation for $c(.,$.$) remains$ true, see section 3. Furthermore the velocity is stratified in the following sense:

$$
\left\{\begin{array}{l}
u(t, x)=u_{b}(t) \times v(t, x), \\
v(., .) \text { is as regular as } c(., .) .
\end{array}\right.
$$

There is no restriction on the isotherms $q_{1}, q_{2}$ for such phenomenons. The only limitation is the local existence of a smooth concentration before shocks.

Thus with a regular concentration (Lipschitz), the main singularity of $u(.,$.$) comes$ from its boundary value and it is a temporal singularity. The structure of the velocity given by (8) is a key element to pass to the weak limit for $u$ and to the strong limit for the concentration. This also allows to propagate high oscillations with large amplitudes for the velocity without affecting the concentration.

Several questions raise up :

First of all, does structure (8) remain true after shock waves? This question concerns the second case (BV concentrations): we try to generalize Structure (8) and its consequences for concentrations $c(.,$.$) in B V$, i.e. for general entropy solutions $(c, u)$ of System $(6)$. For the realistic case with shock-waves, we restrict ourselves to the classical treatment of hyperbolic systems. That is to say, we assume that each eigenvalue is either linearly degenerate or genuinely nonlinear. This restriction implies some convex assumptions on the isotherms $q_{1}, q_{2}$. Nevertheless, this limitation allows us to use the classical Front Tracking Algorithm (FTA see [13]), which gives new estimates for the velocity. For instance, if the boundary velocity belongs to $B V$ with respect to time, the velocity has the same smoothness. Furthermore we obtain better interaction estimates when the shock and rarefaction curves are monotonic in coordinates $(c, \ln u)$. This is the case for instance for an inert gas and an active gas with the Langmuir isotherm.

Above all, we prove that $u / u_{b}$ is $B V$ with respect to time and space with only $\ln \left(u_{b}\right) \in L^{\infty}$ and $c_{0}, c_{b} \in B V$. With this new estimate for the velocity, again we get the stratified Structure (8). This decomposition of the velocity yields a strong stability in $L^{1}$ for concentration. For general weak entropy solutions of quasilinear 
System (6), high oscillations with large amplitude for the velocity can propagate as in semilinear systems, with a strong profile for $u$ with double scale as in [27], see for instance $[25,26,28]$. We conjecture that our result is still valid for general isotherms without any convex restriction.

The second question is : why does the velocity of a system like System (6) has such a simple structure as in System(8)? There are two fundamental reasons to explain this. First, System (6) has a constant zero eigenvalue, which is linearly degenerate ; second, this nonlinear system is linear with respect to $u$.

The system expounded in [4] also has a null eigenvalue and an evident stratified component, but unlike in [4], we cannot reduce System (6) to a single equation in the case of solutions with shocks. P. Bagnerini and M. Rascle ([3]) studied another interesting system, with linearly degenerate eigenvalues, which modelises some traffic flows, but it is a Temple system $([40,41])$. As shown in $[18,19,17,16,31]$, the zero eigenvalue makes the existence of stratified solutions or the propagation of large-amplitude high frequency waves possible. For genuinely nonlinear conservation laws, it is the opposite, since only high oscillating solutions with small amplitude can propagate, see $[23,15]$. Moreover, the linearity of System (6) with respect to the velocity, helps to pass to the weak limit for $u$ and to obtain a strong stability result for the concentration. Furthermore, entropies are also linear with respect to $u$, see section 2 .

As this system has strong features, the third question is, wether it is a Temple system or not. Generally it is not, see section 2.2 for some comments and [12] for a detailed study about the intersection beetween the Temple class and System (6). In [12] we construct an entropy solution with $L^{\infty}$ data exhibiting a blow up. Obviously, results from [5] show that such a blow up is impossible for a Temple system.

The paper is organized as follows: In section 2 we recall some basic results from [10], concerning hyperbolicity, entropies and weak entropy solutions of System (6). In section 3 , we study the case where the concentration is smooth and the velocity is only $L^{\infty}$. In the remainder of the paper we study the case with only $B V$ concentrations. In section 4, we briefly expound the Front Tracking Algorithm (FTA) for System (6). Section 5 is devoted to the study of both shock and rarefaction curves. We state the assumptions that we need to perform estimates with the Front Tracking Algorithm. These assumptions restrict us to convex (or concave) isotherms and we give some examples coming from the chemistry. We show the fundamental interaction estimates in section 6 and $B V$ estimates for $v$ in section 7 . Finally, we obtain strong stability for concentration with respect to weak limits of the boundary velocity in section 8 .

2. Hyperbolicity and entropies. In order for this paper to be self contained, we recall without any proof some results expounded in [10].

It is well known that it is possible to analyze the chromatography system, and thus System (6), in terms of a PDE hyperbolic system, provided we swap time and space variables and $u>0$, see for intance [34] and [36]. In this framework the vector state will be $U=\left(\begin{array}{l}u \\ m\end{array}\right)$ where $m=u c$ is the flow rate of the first species. In this vector state, $u$ must be understood as $u \rho$, that is the total flow rate.

In the sequel, we will use the function $f=q_{1} c_{2}-q_{2} c_{1}$ introduced by Douglas and al. in [29], written here under the form

$$
f(c)=q_{1} c_{2}-q_{2} c_{1}=q_{1}(c)-c h(c) .
$$

Any equilibrium isotherm related to a given species is always increasing with respect to the corresponding concentration (see [29]) i.e. $\frac{\partial q_{i}^{*}}{\partial c_{i}} \geq 0$. Since $c=c_{1}$ and $c_{2}=1-c$, 
we get:

$$
q_{1}^{\prime} \geq 0 \geq q_{2}^{\prime}
$$

Let us define the function $H$ by

$$
H(c)=1+(1-c) q_{1}^{\prime}(c)-c q_{2}^{\prime}(c)=1+q_{1}^{\prime}(c)-c h^{\prime}(c) .
$$

From (10), $H$ satisfies $H \geq 1$ and we have the following relation between $f, H$ and $h$ :

$$
f^{\prime \prime}(c)=H^{\prime}(c)-h^{\prime}(c)
$$

2.1. Hyperbolicity. Concerning hyperbolicity, we refer to [21, 38, 39]. System (6) takes the form

$$
\partial_{x} U+\partial_{t} \Phi(U)=0 \text { with } U=\left(\begin{array}{l}
u \\
m
\end{array}\right) \text { and } \Phi(U)=\left(\begin{array}{c}
h(m / u) \\
I(m / u)
\end{array}\right) .
$$

The eigenvalues are:

$$
0 \text { and } \lambda=\frac{H(c)}{u}
$$

thus, considering (11) the system is strictly hyperbolic. The zero eigenvalue is of course linearly degenerate. Moreover the right eigenvector $r=\left(\begin{array}{c}h^{\prime}(c) \\ 1+q_{1}^{\prime}(c)\end{array}\right)$ associated to $\lambda$ satisfies $d \lambda \cdot r=\frac{H(c)}{u^{2}} f^{\prime \prime}(c)$, so $\lambda$ is genuinely nonlinear in each domain where $f^{\prime \prime} \neq 0$.

Proposition 2.1 ([10] Riemann invariants). System (6) admits the two Riemann invariants:

$c \quad$ and $\quad w=\ln (u)+g(c)=L+g(c), \quad$ where $g^{\prime}(c)=\frac{-h^{\prime}(c)}{H(c)} \quad$ and $L=\ln (u)$.

Furthermore this system can be rewritten for smooth solutions as:

$$
\partial_{x} c+\frac{H(c)}{u} \partial_{t} c=0, \quad \partial_{x}(\ln (u)+g(c))=\partial_{x} w=0
$$

2.2. Entropies. Dealing with entropies, it is more convenient, as shown in [10], to work with the functions

$$
G(c)=\exp (g(c)), \quad W=\exp (w)=u G(c) .
$$

Notice that $G$ is a positive solution of $H G^{\prime}+h^{\prime} G=0$.

Let $E(c, u)$ be any smooth entropy and $Q=Q(c, u)$ any associated entropy flux. Then, for smooth solutions, $\partial_{x} E+\partial_{t} Q=0$. Moreover:

Proposition 2.2 ([10] Representation of all smooth entropies). The smooth entropy functions for System (6) are given by

$$
E(c, u)=\phi(w)+u \psi(c)
$$


where $\phi$ and $\psi$ are any smooth real functions. The corresponding entropy fluxes satisfy

$$
Q^{\prime}(c)=h^{\prime}(c) \psi(c)+H(c) \psi^{\prime}(c) .
$$

In [8], the authors looked for convex entropies for System (12) (i.e. System (6) written in the $(u, m)$ variables) in order to get a kinetic formulation. The next proposition gives us a family of degenerate convex entropies independently of a convex assumption on the function $f$ or on the isotherms.

Proposition 2.3 ([10] Existence of degenerate convex entropies). If $\psi$ is convex or degenerate convex, i.e. $\psi^{\prime \prime} \geq 0$, then $E=u \psi(c)$ is a degenerate convex entropy.

There are some few cases (water vapor or ammonia for instance) where the isotherm is convex. There is also the important case with an inert carrier gas and an active gas with a concave or convex isotherm (see $[9,10,11])$. In these cases, the next proposition ensures the existence of $\lambda$-Riemann invariants which are also strictly convex entropies. In such cases, $w$ is monotonic with respect to $x$ for any entropy solution.

Proposition 2.4 ([10] When $\lambda$-Riemann invariant is a convex entropy). There are strictly convex entropies of the form $E=\phi(w)$ if and only if $G^{\prime \prime}$ does not vanish. More precisely, for $\alpha>0, E_{\alpha}(c, u)=u^{\alpha} G^{\alpha}(c)$ is an increasing entropy with respect to the Riemann invariant $W$. It is strictly convex for $\alpha>1$ if $G^{\prime \prime}>0$ and for $\alpha<1$ if $G^{\prime \prime}<0$.

Unfortunately, a system where $\mathrm{G}$ has an inflexion point does not admit any strictly convex entropy. This is always the case for an inert gas, if the sign of the second derivative of the isotherm changes, see [10] for the BET isotherm.

$w$ is a Riemann invariant associated with a linearly degenerate eigenvalue, namely 0 , so through a contact discontinuity $\partial_{x} w=0$ (see for instance Theorem 8.5.2 page 223 from [21], the reader can also check it directly). Thus through a contact discontinuity, $\partial_{x} \phi(w)=0$ for any $\phi$, without any convexity hypothesis. Does this equality remain valid through a $\lambda$ - shock? When $\lambda$ is genuinely nonlinear ( $f^{\prime \prime}$ does not vanish), the answer is affirmative if System (6) is a Temple system $([40,41,12])$. We recall that a $2 \times 2$ strictly hyperbolic system is a Temple system if there exists a system of coordinates consisting of Riemann invariants and if shock and rarefaction curves coincide. For instance, System (6) with two linear isotherms is a Temple system. More generally System (6) is a Temple system if $\partial_{x} w=0$ through any $\lambda$-shock for any entropy solution. Indeed, the fact that level curves of $w$ are the rarefaction curves and that $\partial_{x} w=0$ through any $\lambda$-shock, means that rarefaction and shock curves coincide. The reader interested in this subject can consult [12], more precisely Lemma 3.1 therein. But unfortunately we have the following remark proven in [12].

REMARK 2.1 ([12]). In general, System (6) is not a Temple system. For an inert gas (for instance the first one: $q_{1}=0$ ) and an active gas with strictly convex isotherm $\left(q_{2}^{\prime \prime}>0\right), P S A$ system is not in the Temple class. It is the case if the active gas is the ammonia or the water vapor. For other examples, see [10].

Proposition 2.5 ([10] Non Existence of strictly convex entropy). If the sign of $G^{\prime \prime}$ changes then System (6) does not admit strictly convex smooth entropy. 
2.3. Definition of weak entropy solution. We have seen that there are two families of entropies: $u \psi(c)$ and $\phi(u G(c))$.

The first family is degenerate convex (in variables $(u, u c)$ ) provided $\psi^{\prime \prime} \geq 0$. So we seek weak entropy solutions which satisfy $\partial_{x}(u \psi(c))+\partial_{t} Q(c) \leq 0$ in the distribution sense.

The second family is not always convex. There are only two interesting cases, namely $\pm G^{\prime \prime}(c)>0$ for all $c \in[0,1]$. When $G^{\prime \prime}>0$ and $\alpha>1$, we expect to have $\partial_{x}(u G(c))^{\alpha} \leq 0$ from Proposition 2.4. But the mapping $W \mapsto W^{\alpha}$ is increasing on $\mathbb{R}^{+}$, so the last inequality reduces to $\partial_{x}(u G(c)) \leq 0$.

In the same way, if $G^{\prime \prime}<0$, we get $\partial_{x}(u G(c)) \geq 0$.

Now we can state a mathematical definition of weak entropy solutions.

Definition 2.1. Let $T>0, X>0, u \in L^{\infty}\left((0, T) \times(0, X), \mathbb{R}^{+}\right), 0 \leq c(t, x) \leq$ $\rho \equiv 1$ for almost all $(t, x) \in(0, T) \times(0, X)$. Then $(c, u)$ is a weak entropy solution of System (6)-(7) with respect to the family of entropies $u \psi(c)$ if, for all convex (or degenerate convex) $\psi$ :

$$
\frac{\partial}{\partial x}(u \psi(c))+\frac{\partial}{\partial t} Q(c) \leq 0,
$$

in $\mathcal{D}^{\prime}\left(\left[0, T\left[\times\left[0, X[)\right.\right.\right.\right.$, where $Q^{\prime}=H \psi^{\prime}+h^{\prime} \psi$, that is, for all $\phi \in \mathcal{D}\left(\left[0, T\left[\times\left[0, X\left[; \mathbb{R}_{+}\right)\right.\right.\right.\right.$:

$$
\begin{aligned}
\int_{0}^{X} \int_{0}^{T}\left(u \psi(c) \partial_{x} \phi+Q(c) \partial_{t} \phi\right) d t d x & +\int_{0}^{T} u_{b}(t) \psi\left(c_{b}(t)\right) \phi(t, 0) d t \\
& +\int_{0}^{X} Q\left(c_{0}(x)\right) \phi(0, x) d x \geq 0
\end{aligned}
$$

REMARK 2.2. If $\pm G^{\prime \prime} \geq 0$ then $u \psi= \pm u G(c)$ is a degenerate convex entropy, with entropy flux $Q \equiv 0$, contained in the family of entropies $u \psi(c)$. So, if $G^{\prime \prime}$ keeps a constant sign on $[0,1],(c, u)$ has to satisfy:

$$
\pm \frac{\partial}{\partial x}(u G(c)) \leq 0, \quad \text { if } \pm G^{\prime \prime} \geq 0 \text { on }[0,1] .
$$

Notice that the entropies $u \psi(c)$ and the entropy $u G(c)$ are linear with respect to the velocity $u$.

2.4. About the Riemann problem. The implementation of the Front Tracking Algorithm used extensively in section 4 requires some results about the solvability of the Riemann problem. We first recall the solution of the boundary Riemann problem, i.e. System (6) with the following data:

$$
c(0, x)=c^{0} \in[0,1], \quad x>0, \quad\left\{\begin{array}{l}
c(t, 0)=c^{+} \in[0,1], \quad t>0 . \\
u(t, 0)=u^{+}>0,
\end{array}\right.
$$

We restrict ourselves to the case $f^{\prime \prime} \neq 0$, thus there is no $\lambda$-discontinuity wave and no composite wave as in [10].

The complete Riemann problem (6), (17)

$$
\left\{\begin{array}{l}
c(t, 0)=c^{-} \in[0,1], \quad t<0,\left\{\begin{array}{rl}
c(t, 0) & =c^{+} \in[0,1], \\
u(t, 0) & =u^{-}>0,
\end{array} \quad t>0 .\right.
\end{array}\right.
$$


is simply solved by a 0 -contact discontinuity wave followed by a $\lambda$-wave given by following propositions (see Fig. 2 below).

For the boundary Riemann problem (6), (16), we are classically looking for a selfsimilar solution, i.e.: $c(t, x)=C(z), u(t, x)=U(z)$ with $z=\frac{t}{x}>0$ (see Fig. 1).

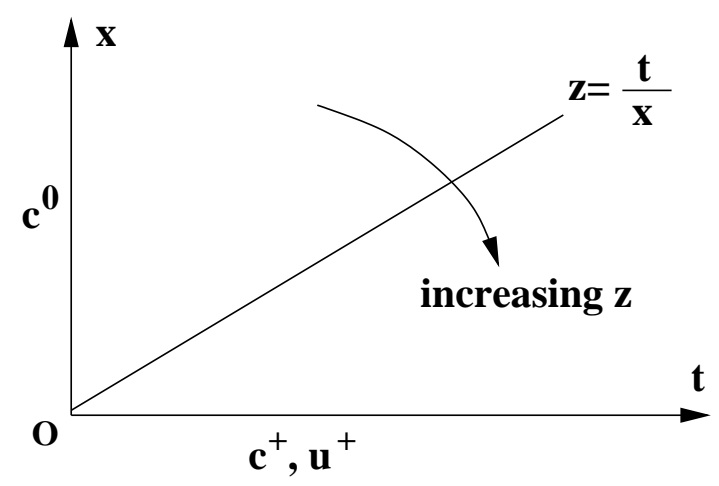

FIG. 1. Data for the boundary Riemann problem

In the domain $t>0, x>0$, the boundary Riemann problem is solved with a $\lambda$-wave since $\lambda$ is the only positive eigenvalue of the system. Let us recall the following results obtained in [10], where $f$ and $H$ are given by $(9,11)$.

Proposition 2.6 ( $\lambda$-rarefaction waves). Any smooth non-constant self-similar solution $(C(z), U(z))$ of $(6)$ in an open domain $\Omega=\{0 \leq \alpha<z<\beta\}$ where $f^{\prime \prime}(C(z))$ does not vanish, satisfies:

$$
\frac{d C}{d z}=\frac{H(C)}{z f^{\prime \prime}(C)}, \quad U(z)=\frac{H(C)}{z} .
$$

In particular, $\frac{d C}{d z}$ has the same sign as $f^{\prime \prime}(C)$.

Assume for instance that $0 \leq a<c^{0}<c^{+}<b \leq 1$ and $f^{\prime \prime}>0$ in $] a, b[$. Then the only smooth self-similar solution of $(6)$ is such that:

$$
\left\{\begin{aligned}
C(z) & = & c^{0}, & 0<z<z_{0}, \\
\frac{d C}{d z} & = & \frac{H(C)}{z f^{\prime \prime}(C)}, & z_{0}<z<z_{+}, \\
C(z) & = & c_{+}, & z_{+}<z
\end{aligned}\right.
$$

where $z^{+}=\frac{H\left(c^{+}\right)}{u^{+}}, z^{0}=z^{+} e^{-\Phi\left(c^{+}\right)}$with $\Phi(c)=\int_{c^{0}}^{c} \frac{f^{\prime \prime}(\xi)}{H(\xi)} d \xi$. Moreover $u^{0}=\frac{H\left(c^{0}\right)}{z^{0}}$ and $U$ is given by:

$$
\left\{\begin{array}{rrrr}
U(z) & = & u_{0}, & 0<z<z_{0} \\
U(z)= & \frac{H(C(z))}{z}, & z_{0}<z<z_{+} \\
U(z)= & u_{+} & z_{+}<z
\end{array}\right.
$$

Proposition 2.7 ( $\lambda$-shock waves). If $\left(c^{0}, c^{+}\right)$satisfies the following admissibility condition, equivalent to the Liu entropy-condition ([30]):

$$
\text { for all } c \text { between } c^{0} \text { and } c^{+}, \quad \frac{f\left(c^{+}\right)-f\left(c^{0}\right)}{c^{+}-c^{0}} \leq \frac{f(c)-f\left(c^{0}\right)}{c-c^{0}},
$$


then the boundary Riemann problem (6), (16) is solved by a shock wave defined as:

$$
C(z)=\left\{\begin{array}{ll}
c^{0} & \text { if } \quad 0<z<s, \\
c^{+} & \text {if } \quad s<z
\end{array}, \quad U(z)= \begin{cases}u^{0} & \text { if } \quad 0<z<s, \\
u^{+} & \text {if } \quad s<z,\end{cases}\right.
$$

where $u^{0}$ and the speed $s$ of the shock are obtained through

$$
\frac{[f]}{u^{0}[c]}+\frac{1+h^{0}}{u^{0}}=s=\frac{[f]}{u^{+}[c]}+\frac{1+h^{+}}{u^{+}}
$$

where $[c]=c^{+}-c^{0},[f]=f^{+}-f^{0}=f\left(c^{+}\right)-f\left(c^{0}\right), h^{+}=h\left(c^{+}\right), h^{0}=h\left(c^{0}\right)$.

A 0 -wave always appears on the line $\{t=0\}$.

Proposition 2.8 (0-contact discontinuity waves). Two distinct states $U^{-}$and $U^{0}$ are connected by a 0 -contact discontinuity if and only if $c^{-}=c^{0}$ (with of course $\left.u^{-} \neq u^{0}\right)$.

In conclusion the solution of the Rieman problem for $x>0$ and a convex function $f$ is

- $(c, u)=\left(c^{-}, u^{-}\right)$for $t<0$,

- a 0 -contact discontinuity for $t=0$,

- a $\lambda$ - wave for $t>0$,

In practice, since $c^{0}=c^{-}$, we first solve the boundary Riemann Problem. Thus $u^{0}$ is well defined and the 0 -contact discontinuity is automatically solved.
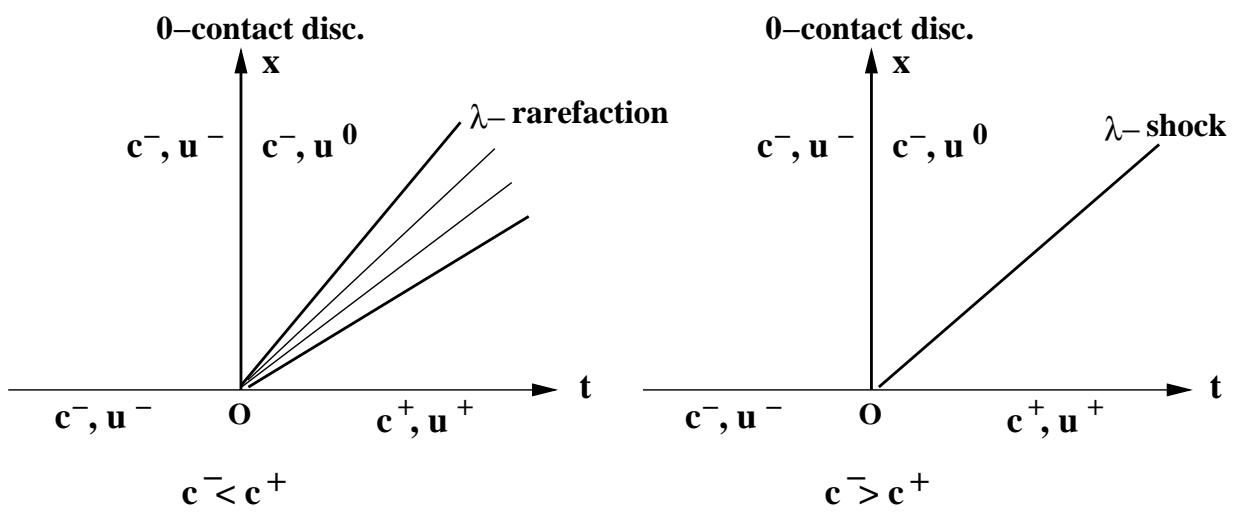

FIG. 2. Solution of the Riemann problem when $f^{\prime \prime}>0$.

3. Case with smooth concentration. System (6) has the strong property that there exist weak entropy solutions with smooth concentration $c(.,$.$) on (0, T) \times(0, X)$ but not necessarily smooth velocity $u(.,$.$) , for some positive constants T$ and $X$. Furthermore, $c(.,$.$) is the solution of a scalar conservation law.$

3.1. Existence of weak entropy solutions with smooth concentration. About existence of weak entropy solutions of some hyperbolic systems with one component less smooth than the others, we refer the reader to $[18,19,31]$. Here, we obtain by the classical method of characteristics existence and uniqueness of weak entropy solutions with smooth concentration and $L^{\infty}$ velocity. We have a similar result in [9] but only with smooth velocity. 
TheOREM 3.1 (Unique weak entropy solution with smooth concentration). Let $T_{0}>0, X>0, c_{0} \in W^{1, \infty}([0, X],[0,1]), c_{b} \in W^{1, \infty}\left(\left[0, T_{0}\right],[0,1]\right), \ln u_{b} \in$ $L^{\infty}\left(\left[0, T_{0}\right], \mathbb{R}\right)$.

If $c_{0}(0)=c_{b}(0)$ then there exists $\left.\left.T \in\right] 0, T_{0}\right]$ such that System (6)-(7) admits a unique weak entropy solution $(c, u)$ on $[0, T] \times[0, X]$ with

$$
c \in W^{1, \infty}([0, T] \times[0, X],[0,1]), \quad \ln u \in L^{\infty}\left([0, T], W^{1, \infty}([0, X], \mathbb{R})\right) .
$$

Furthermore, for any $\psi \in C^{1}([0,1], \mathbb{R})$, setting

$$
F^{\prime}(c)=(H(c) G(c))^{-1} \text { and } Q^{\prime}=H \psi^{\prime}+h^{\prime} \psi,
$$

(c,u) satisfies:

$$
\begin{array}{r}
\partial_{x}(u \psi(c))+\partial_{t} Q(c)=0, \quad \partial_{x}(u G(c))=0, \\
\partial_{t} c+u_{b}(t) G\left(c_{b}(t)\right) \partial_{x} F(c)=0 .
\end{array}
$$

Equations (21) mean that entropy inequalities become equalities. This fact easily implies the stratified structure (8) of the velocity, see equation (23) below. Notice that system (6) degenerates into scalar equation (22).

Proof. We build a solution using the Riemann invariants and we check that such a solution is an entropy solution. Next, we prove uniqueness.

Using the Riemann invariant $W=u G(c)\left(\partial_{x} W=0\right)$ and the boundary data, we define $u$ by:

$$
u(t, x)=\frac{u_{b}(t) G\left(c_{b}(t)\right)}{G(c(t, x))}
$$

so $u$ is smooth with respect to $x$. Then, the first equation of (13) can be rewritten as follows:

$$
\partial_{t} c+\mu \partial_{x} c=0, \quad \text { with } \quad \mu=\lambda^{-1}=\frac{u}{H(c)}=\frac{u_{b}(t) G\left(c_{b}(t)\right)}{H(c) G(c)}=\mu(t, c) .
$$

We solve (24), supplemented by initial-boundary value data $\left(c_{0}, c_{b}\right)$ by the standard characteristics method. For a given $(\tau, x)$, let us define, $X(\cdot, \tau, x)$ as the solution of:

$$
\frac{d X(s, \tau, x)}{d s}=\mu(s, c(s, X(s, \tau, x))), \quad X(\tau, \tau, x)=x .
$$

Since $\frac{d c}{d s}(s, X(s, \tau, x))=0$, from $(24)$ we have

$$
X(s, \tau, x)=x-b(s, \tau) F^{\prime}(c(\tau, x)) \text { with } b(s, \tau)=\int_{s}^{\tau} u_{b}(\sigma) G\left(c_{b}(\sigma)\right) d \sigma .
$$

Now, for some $T \in\left[0, T_{0}\right]$ defined later on, we split $\Omega=[0, T] \times[0, X]$ according to the characteristic line $\Gamma$ issuing from the corner $(0,0)$, i.e. we define the sets $\Omega^{ \pm}=\{(t, x) \in \Omega, \pm(x-X(t, 0,0)) \geq 0\}$.

Since $\partial_{x} X(t, 0, x)=1-b(t, 0) F^{\prime \prime}\left(c_{0}(x)\right) \partial_{x} c_{0}(x), b(0,0)=0$ and $b(., 0) \in W^{1, \infty}\left(\Omega^{+}\right)$, 
the mapping $x \mapsto X(t, 0, x)$ is a Lipschitz diffeomorphism for $0 \leq t \leq T$ with $T \in] 0, T_{0}$ ] small enough. Then we define $\xi(t, x)$ on $\Omega^{+}$: for each $t \in[0, T], X(t, 0, \xi(t, x))=x$. Then we have $c(t, x)=c_{0}(\xi(t, x))$ on $\Omega^{+}$. Furthermore $\partial_{t} \xi=-\partial_{s} X / \partial_{x} X$ and thus $c$ is Lipschitz continuous in time and space on $\Omega^{+}$.

We work in a similar way on $\Omega^{-}$and $c \in W^{1, \infty}\left(\Omega^{-}\right)$. Since $c$ is continuous on $\Gamma$ from the compatibility conditions $c_{0}(0)=c_{b}(0)$, we have $c \in W^{1, \infty}(\Omega)$.

By construction $(c, u)$ satisfies (13), rewritten as follows:

$$
\partial_{x} \ln u=-\partial_{x} g(c), u \partial_{x} c+H \partial_{t} c=0 .
$$

These equations imply:

$$
\partial_{x} u=-u \partial_{x} g(c)=-u g^{\prime}(c) \partial_{x} c=-u g^{\prime}(c)\left(-\frac{H(c)}{u} \partial_{t} c\right)=-h^{\prime}(c) \partial_{t} c=-\partial_{t} h(c) .
$$

Now we check that $(c, u)$ satisfies $(21)$. Let $\psi$ be a $C^{1}$ function. Using the identity $Q^{\prime}=h^{\prime} \psi+H \psi^{\prime}$, the previous equations and the differentiability almost everywhere of $u$ with respect to $x$, we have:

$$
\partial_{x}(u \psi(c))+\partial_{t} Q(c)=\psi \partial_{x} u+u \psi^{\prime} \partial_{x} c+Q^{\prime} \partial_{t} c
$$

$$
=\psi\left(\partial_{x} u+h^{\prime} \partial_{t} c\right)+\psi^{\prime}\left(u \partial_{x} c+H \partial_{t} c\right)=\psi \times 0+\psi^{\prime} \times 0=0 .
$$

Obviously, (21) implies (14), so $(c, u)$ is an entropy solution of System (6).

We now prove the uniqueness of such a weak entropy solution.

Precisely, if both $c \in W^{1, \infty}([0, T] \times[0, X],[0,1])$ and $\ln u \in L^{\infty}\left((0, T), W^{1, \infty}(0, X)\right)$ satisfy (14) in $\mathcal{D}^{\prime}\left(\left[0, T\left[\times\left[0, X[)\right.\right.\right.\right.$ with initial-boundary data $c_{0}, c_{b}, u_{b}$, then we show that $(c, u)$ is necessarily the previous solution built by the characteristics' method.

Choosing the convex functions $\psi(c)= \pm 1$ and $\psi(c)= \pm c$ we obtain (6). The main element to achieve the proof is the fact that $u$ admits almost everywhere a classical partial derivative with respect to $x$. Thus we use the classical chain rule formula which allows to obtain (13) with smooth functions, see the proof of Proposition 2.1 from [9]. Indeed, computations are similar as in (25) where the chain rule formula is valid almost everywhere.

Now $(c, u)$ satisfies (13), which implies, using the beginning of the proof of Theorem 3.1 , that $(c, u)$ is necessarily our previous solution.

REMARK 3.1.

1. Notice that $T, X$ are only depending on $\left\|\ln \left(u_{b}\right)\right\|_{L^{\infty}},\left\|c_{b}\right\|_{W^{1, \infty}},\left\|c_{0}\right\|_{W^{1, \infty}}$. Thus, if $\left(u_{b}^{\varepsilon}\right)_{0<\varepsilon<1}$ is a sequence of boundary velocity data such that $\left(\ln u_{b}^{\varepsilon}\right)$ is uniformly bounded in $L^{\infty}\left(0, T_{0}\right)$, and if $\left(c_{0}^{\varepsilon}\right),\left(c_{b}^{\varepsilon}\right)$ are some initial and boundary concentration data uniformly bounded in $W^{1, \infty}$ with the compatibility condition at the corner $c_{0}^{\varepsilon}(0)=c_{b}^{\varepsilon}(0)$, then there exist $T>0$ and $X>0$ and Lipschitz bounds for $c^{\varepsilon}, \ln u^{\varepsilon}$ on $[0, T] \times[0, X]$ independent of $\varepsilon$.

2. As in [9], we have a global solution with smooth concentration if $\lambda$ is genuinely nonlinear (for instance an inert gas case and a Langmuir isotherm), with monotonic assumptions on $c_{0}$ and $c_{b}$.

3.2. Strong stability with respect to velocity. In the case of a Lipschitz continuous concentration, we now give a strong stability result for the concentration with respect to a weak limit of the boundary velocity.

Theorem 3.2 (Strong stability for smooth concentration). Let $T_{0}>0, X>$ $0, c_{0} \in W^{1, \infty}([0, X],[0,1]), c_{b} \in W^{1, \infty}\left(\left[0, T_{0}\right],[0,1]\right)$ such that $c_{0}(0)=c_{b}(0)$, and 
$\left(\ln u_{b}^{\varepsilon}\right)_{0<\varepsilon<1}$ a bounded sequence in $L^{\infty}\left(0, T_{0}\right)$. Then, there exists $\left.T \in\right] 0, T_{0}[$ such that System (6) admits a unique weak entropy solution $\left(c^{\varepsilon}, u^{\varepsilon}\right)$ with $c^{\varepsilon} \in W^{1, \infty}([0, T] \times$ $[0, X],[0,1]), \ln u^{\varepsilon} \in L^{\infty}\left([0, T], W^{1, \infty}([0, X], \mathbb{R})\right)$ and with initial and boundary values:

$$
\left\{\begin{array}{l}
c^{\varepsilon}(0, x)=c_{0}(x) \in[0,1], \quad x>0 \\
c^{\varepsilon}(t, 0)=c_{b}(t) \in[0,1], \quad t>0 \\
u^{\varepsilon}(t, 0)=u_{b}^{\varepsilon}(t)>0, \quad t>0 .
\end{array}\right.
$$

If $\left(u_{b}^{\varepsilon}\right)$ converges towards $\bar{u}_{b}$ in $L^{\infty}\left(0, T_{0}\right)$ weak-* when $\varepsilon$ goes to 0 , then $\left(c^{\varepsilon}\right)$ converges in $L^{\infty}([0, T] \times[0, X])$ towards the unique smooth solution of

$$
\partial_{t} c+\bar{u}_{b}(t) G\left(c_{b}(t)\right) \partial_{x} F(c)=0, \quad c(t, 0)=c_{b}(t), c(0, x)=c_{0}(x) .
$$

Furthermore we have:

$$
\lim _{\varepsilon \rightarrow 0}\left\|u^{\varepsilon}(t, x)-u_{b}^{\varepsilon}(t) \frac{G\left(c_{b}(t)\right)}{G(c(t, x))}\right\|_{L^{\infty}([0, T] \times[0, X])}=0 .
$$

Proof. Thanks to Theorem 3.1 and Remark 3.1 1., there exists $T>0$ such that System (6), with initial and boundary values (26) admits the unique weak entropy solution $\left(c^{\varepsilon}, u^{\varepsilon}\right)$, with smooth concentration in the previous sense on $[0, T] \times[0, X]$. Since $\left(c^{\varepsilon}\right)$ is bounded in $W^{1, \infty}$, up to a subsequence, $\left(c^{\varepsilon}\right)$ converges strongly in $L^{\infty}$ to c. Using (22) in conservative form, we can pass to the limit and get (27). Problem (27) has a unique solution using the characteristics' method. Thus, the whole sequence $\left(c^{\varepsilon}\right)$ converges. We recover the last limit for $u^{\varepsilon}$ thanks to $\partial_{x}\left(u^{\varepsilon} G\left(c^{\varepsilon}\right)\right)=0$.

Notice that if $\bar{u}_{b}$ is a constant function, for instance $u_{b}^{\varepsilon}(t)=u_{b}(t / \varepsilon)$ with $u_{b}$ periodic, we can compute the concentration using only a constant velocity (the mean velocity) as in liquid chromatography.

$\underline{\text { An example from geometric optics: }} \quad$ if $u_{b}^{\varepsilon}(t)=u_{b}\left(t, \frac{t}{\varepsilon}\right)$ where $u_{b}(t, \theta) \in$ $L^{\infty}\left((0, T), C^{0}(\mathbb{R} / \mathbb{Z})\right)$ and $\inf u_{b}>0$, we have a similar result with Equation $(27)$ for $c(.,$.$) where \bar{u}_{b}(t)=\int_{0}^{1} u_{b}(t, \theta) d \theta$ and a profile $U$ :

$$
\lim _{\varepsilon \rightarrow 0}\left\|u^{\varepsilon}(t, x)-U\left(t, x, \frac{t}{\varepsilon}\right)\right\|_{L^{\infty}}=0 \text { where } U(t, x, \theta)=u_{b}(t, \theta) \frac{G\left(c_{b}(t)\right)}{G(c(t, x))} .
$$

Other examples can be found in $[17,16]$.

4. Front tracking algorithm. In Section 3, where $c$ is smooth and $\ln u_{b}$ is in $L^{\infty}(0, T)$, we have seen that there exists a stratified structure for the velocity: $u(t, x)=u_{b}(t) v(t, x)$, see (8). Furthermore $c$ satisfies the scalar conservation law $(22)$. For only $B V$ data we cannot expect to obtain such a scalar conservation law for the concentration, except in the case of linear isotherms. In that case, the scalar conservation law (22) and System (6) have the same solution for the Riemann problem, but linear isotherms are of poor interest from a chemical engineering point of view. The first interesting case is the case with an inert gas and a Langmuir isotherm, which was mathematically studied for the first time in [9].

Nevertheless we guess that (8) is still true with $v \in B V$. From $[9,10]$ we have already obtained $B V$ regularity with respect to $x$ with a Godunov scheme. To get 
$B V$ regularity with respect to $t$ we will use a more precise algorithm to study wave interactions, namely a Front Tracking Algorithm (FTA).

The Front Tracking method for scalar conservation laws was introduced by Dafermos, [20]. The method was extended to genuinely nonlinear systems of two conservation laws by DiPerna [22]. For our purpose, we do not use the generalization to genuinely nonlinear systems of any size by Bressan [13] or Risebro [35].

The FTA is much more complicated when an eigenvalue is piecewise genuinely nonlinear, see $[2,1,24]$. This is why, we restrict ourselves to the case where $\lambda$ is genuinely nonlinear, which allows us to treat some relevant cases from the point of view of chemical engineering, like an inert gas with a Langmuir isotherm, or two active gases with a binary Langmuir isotherm. For this purpose we work in the framework expounded in the recent and already classical Bressan's Book [14]. In this framework we assume $f^{\prime \prime} \geq 0$, thus a Riemann problem presents only two waves:

1. a contact discontinuity with speed 0 ,

2. a rarefaction wave with speed $\lambda>0$ or a shock wave with speed between $\lambda^{-}$ and $\lambda^{+}$, characteristic speeds associated to the left and right states, respectively.

Let $\delta>0$. A $\delta$-approximate Front Tracking solution of System (6) is a pair of piecewise constant functions $c^{\delta}(t, x), u^{\delta}(t, x)$, whose jumps are located along a finite number of straight lines $t=t_{\alpha}(x)$ in the $t-x$ plane and approximately satisfy the entropy conditions. For each $x>0$ and $\psi^{\prime \prime} \geq 0$, one should thus have an estimate of the form:

$$
\sum_{\alpha}\left(\left[u^{\delta} \psi\left(c^{\delta}\right)\right]-\frac{d t_{\alpha}}{d x}\left[Q\left(c^{\delta}\right)\right]\right)\left(t_{\alpha}, x\right) \leq \mathcal{O}(\delta),
$$

where $[u]=u^{+}-u^{-}$is the jump across a jump line, and the sum is taken over all jump for $x$ fixed. Inequality (28) implies that $\left(c^{\delta}, u^{\delta}\right)$ is "almost an entropy solution":

$$
\partial_{x}\left(u^{\delta} \psi\left(c^{\delta}\right)\right)+\partial_{t} \psi\left(c^{\delta}\right) \leq \mathcal{O}(\delta) .
$$

That's enough to get an entropy solution "issued from FTA" when $\delta$ tends to zero.

Since we only want to use piecewise constant functions, it is convenient to approximate a continuous rarefaction wave by a piecewise constant function. For this purpose, the rarefaction curve is discretized with a step of order $\delta$ and thus (28) still holds.

We now briefly describe an algorithm which generates these Front Tracking approximations. The construction starts on the initial line $x=0$ and the boundary $t=0$, by taking a piecewise constant approximation of the initial value $c_{b}(t), u_{b}(t)$ and boundary values $c_{0}(x)$. Let $t_{1}<\cdots<t_{N}, \tilde{x}_{1}<\cdots<\tilde{x}_{M}$ be the points where initial-boundary values are discontinuous. For each $\alpha=1, \cdots, N$, the Riemann problem generated by the jump of initial constant values at $\left(t_{\alpha}, x=0\right)$ is approximately solved on a forward neighbourhood of $\left(t_{\alpha}, 0\right)$ in the $t-x$ plane, by a function invariant on line $t-t_{\alpha}=\mathrm{a} x$, for all positive $a$, and piecewise constant. Notice that the boundary is characteristic, thus we only have one wave associated with the speed $\lambda$ in the corner $(0,0)$.

The approximate solution $\left(c^{\delta}, u^{\delta}\right)$ can then be prolonged until $x_{1}>0$ is reached, when the first set of interactions between two wave-fronts takes place. If $x_{1}>\tilde{x}_{1}$ we first have to solve the characteristic boundary Riemann problem at $\left(t=0, x=\tilde{x}_{1}\right)$. Since $\left(c^{\delta}, u^{\delta}\right)\left(., x_{1}\right)$ is still a piecewise constant function, the corresponding Riemann problems can again be approximately solved within the class of piecewise constant 
functions. The solution is then continued up to a value $x_{2}$ where the next characteristic boundary Riemann problem occurs or the second set of wave interactions takes place, and so on.

According to this algorithm, contact discontinuity fronts travel with speed zero, shock fronts travel exactly with Rankine-Hugoniot speed, while rarefaction fronts travel with an approximate characteristic speed. However, one exception to this rule must be allowed if three or more fronts meet at the same point. To avoid this situation, we must change the positive speed $\lambda$ of one of the incoming shock fronts or rarefaction fronts. Of course this change of speed can be chosen arbitrarily small and we have again Inequality (28).

Notice that, for $2 \times 2$ system the number of wave-fronts cannot approach infinity in finite $x>0$. DiPerna shows in [22] that the process of regenerating the solution by solving local Riemann problems yields an approximating solution within the class of piecewise constant functions that is globally defined and that contains only a finite number of discontinuities in any compact subset of the $t-x$ quarter plane $t \geq 0, x \geq 0$. We then do not consider non-physical fronts as in [14] for general $n \times n$ systems with $n \geq 3$.

5. About the shock and rarefaction curves. In this section, we present the necessary hypothesis to use the FTA with large data. Precisely we work in the classical hyperbolic case, namely, eigenvalues are linearly degenerate or genuinely nonlinear. We assume that:

$$
\lambda=\frac{H(c)}{u} \text { is genuinely nonlinear } \quad \text { i.e } f \text { is convex on }[0,1] .
$$

Actually $\lambda$ is genuinely nonlinear for $f^{\prime \prime} \neq 0$, but since $f=c_{1} q_{2}-c_{2} q_{1}$ (see (9)), we can assume that $f^{\prime \prime}>0$, exchanging the gas indexes if necessary.

Our analysis of wave interactions in Section 6 is more precise with monotonic $\lambda$-wave curves, then we also assume:

$$
\lambda \text {-wave curves are monotonic. }
$$

To state precisely this last assumption, let us introduce some notations. Let $\left(c_{-}, L_{-}\right)$ be a left constant state, connected to $\left(c_{+}, L_{+}\right)$, a right constant state, by a $\lambda$-wave curve. In the genuinely linear case, with Assumption (30), $\lambda$-wave curve is a rarefaction curve with $c_{-}<c_{+}$or a shock curve with $c_{-}>c_{+}$. The sign of $[c]=c_{+}-c_{-}$ comes from the general study of the Riemann problem in [10]. From the Riemann invariant $w=\ln u+g(c)$ and the Rankine-Hugoniot conditions, a $\lambda$-wave curve can be written as follows (see [10]):

$$
\begin{aligned}
{[L]=L_{+}-L_{-}=\ln u_{+}-\ln u_{-} } & =T\left(c_{+}, c_{-}\right) \\
& = \begin{cases}-[g]=-\left(g\left(c_{+}\right)-g\left(c_{-}\right)\right) & \text {if } c_{-}<c_{+} \\
S\left(c_{+}, c_{-}\right) & \text {else. }\end{cases}
\end{aligned}
$$

We give an explicit formula for $S$ in Lemma 5.1.

Notice that we only use one Riemann invariant, namely $c$, to write $\lambda$-wave curves. Indeed $L=\ln u$ and $c$ have quite different behavior as seen in $[9,10]$ and in this paper. Furthermore we can give some simple criterion to have monotonic $\lambda$-wave curves. For instance, as $g^{\prime}=-h^{\prime} / H$, the rarefaction curve is monotonic if and only if $h$ is monotonic. A chemical example, investigated in [9], is the case of an inert gas 
$\left(q_{1}=0\right)$ and an active gas with a Langmuir isotherm: $q_{2}^{*}\left(c_{2}\right)=Q_{2} \frac{K_{2} c_{2}}{1+K_{2} c_{2}}$. In this case we have

$$
f^{\prime \prime}>0, \quad h^{\prime}<0, \quad \frac{\partial S}{\partial c_{-}} \geq 0 \geq \frac{\partial S}{\partial c_{+}} .
$$

The first condition of (33) gives us (30) and the last one gives us (31). Notice that if we exchange gas indexes, Inequalities (33) simply become:

$$
f^{\prime \prime}<0<h^{\prime}, \quad \frac{\partial S}{\partial c_{-}} \leq 0 \leq \frac{\partial S}{\partial c_{+}} .
$$

Let us give some isotherm examples which satisfy (30) and (31) .

Proposition 5.1. In the following examples, Assumptions (30), (31) are valid:

1. one gas is inert: $q_{1}=0$, and the other has a concave isotherm: $q_{2}^{\prime \prime} \leq 0$,

2. two active gases with linear isotherms: $q_{i}^{*}\left(c_{1}, c_{2}\right)=K_{i} c_{i}, i=1,2$,

3. two active gases with binary Langmuir isotherms: $q_{i}^{*}\left(c_{1}, c_{2}\right)=$ $\frac{Q_{i} K_{i} c_{i}}{1+K_{1} c_{1}+K_{2} c_{2}}, i=1,2$, where positive constants $Q_{1}, Q_{2}, K_{1} \geq K_{2}$ satisfy: $Q_{1} K_{1}<Q_{2} K_{2}$.

Furthermore, for two active gases with binary Langmuir isotherms, $\lambda$ is genuinely nonlinear, i.e. (30) is satisfied, if $Q_{1} K_{1} \neq Q_{2} K_{2}$.

The first case is the most classical case, where only one gas is active and its isotherm has no inflexion point, like with the Langmuir isotherm.

The second case is less interesting in chemistry and only valid when the concentrations are near a constant state.

For the third case, notice that $K_{1} \geq K_{2}$ is not really an assumption (exchange the indexes if necessary).

Proof of Proposition 5.1. We use some technical Lemmas postponed to Subsection 5.1. The point is to satisfy (33).

1. Case with an inert gas: we have $h=q_{2}, f(c)=-c h(c), f^{\prime}=-h-c h^{\prime}, f^{\prime \prime}=$ $-2 h^{\prime}-\overline{c h^{\prime \prime} \text {, which implies } h^{\prime}}=q_{2}^{\prime} \leq 0, h^{\prime \prime}=q_{2} " \leq 0$ and then $f^{\prime \prime} \geq 0$. We conclude thanks to Lemmas 5.3 and 5.4 .

2. Case with linear isotherms: linear isotherms are $q_{1}(c)=K_{1} c, q_{2}(c)=K_{2}(1-c)$ with $K_{1} \geq 0, K_{2} \geq 0$ then $q_{1}^{\prime}(c)=K_{1} \geq 0, q_{2}^{\prime}(c)=-K_{2} \leq 0, h^{\prime}(c)=q_{1}^{\prime}(c)+q_{2}^{\prime}(c)=$ $K_{1}-K_{2}, f^{\prime \prime}(c)=2\left(K_{2}-K_{1}\right)$. We assume $K_{1} \leq K_{2}$, then we have $h^{\prime} \leq 0 \leq f^{\prime \prime}$. Since $q_{i} "=0, i=1,2$, we conclude thanks to Lemmas 5.3 and 5.5.

3. Case with a binary Langmuir isotherm: we have $q_{1}(c)=\frac{Q_{1} K_{1} c}{D}, q_{2}(c)=$ $\frac{Q_{2} K_{2}(1-c)}{D}$ where $D=1+K_{1} c+K_{2}(1-c)$. Then $q_{1}^{\prime}(c)=\frac{Q_{1} K_{1}\left(1+K_{2}\right)}{D^{2}} \geq$ $0, q_{2}^{\prime}(c)=-\frac{Q_{2} K_{2}\left(1+K_{1}\right)}{D^{2}} \leq 0$,

$h^{\prime}(c)=q_{1}^{\prime}(c)+q_{2}^{\prime}(c) \leq 0$ if and only if $Q_{1} K_{1}\left(1+K_{2}\right) \leq Q_{2} K_{2}\left(1+K_{1}\right)$,

$q_{1}^{\prime \prime}(c)=\frac{2 Q_{1} K_{1}\left(1+K_{2}\right)\left(K_{2}-K_{1}\right)}{D^{3}} \leq 0$ if and only if $K_{1} \geq K_{2}$,

$q_{2}^{\prime \prime}(c)=\frac{2 Q_{2} K_{2}\left(1+K_{1}\right)\left(K_{1}-K_{2}\right)}{D^{3}} \geq 0$ if and only if $K_{1} \geq K_{2}$, 
$f^{\prime \prime}(c)=\frac{2\left(Q_{2} K_{2}-Q_{1} K_{1}\right)\left(1+K_{1}\right)\left(1+K_{2}\right)}{D^{3}} \geq 0$ if and only if $Q_{2} K_{2} \geq Q_{1} K_{1}$.

Since $Q_{1} K_{1} \leq Q_{2} K_{2}$, we get $f^{\prime \prime} \geq 0$ and $\frac{Q_{1}}{Q_{2}} \leq \frac{K_{2}}{K_{1}} .1 \leq \frac{1+K_{1}}{1+K_{2}}$ because $K_{1} \geq K_{2}$, so we have $\frac{Q_{1}}{Q_{2}} \leq \frac{K_{2}}{K_{1}} \frac{1+K_{1}}{1+K_{2}}$, i.e. $h^{\prime} \leq 0$. Now we conclude with Lemmas 5.3 and 5.5.

5.1. Technical lemmas about shock curves. We express the shock curves as follows.

Lemma 5.1. We have $\exp \left\{S\left(c_{+}, c_{-}\right)\right\}=\frac{u_{-}}{u_{+}}=\frac{\alpha+h_{-}}{\alpha+h_{+}}$, where $h_{ \pm}=h\left(c_{ \pm}\right)$and $\alpha=\frac{[f]}{[c]}+1$.

Proof. First, from the Rankine Hugoniot conditions: $\frac{[u c]}{\left[c+q_{1}(c)\right]}=\frac{[u]}{[h]}$, i.e. $[h]=$ $\frac{[u]\left[c+q_{1}(c)\right]}{[u c]}$, we obtain

$$
\frac{u_{+}}{u_{-}}=\frac{\left[c+q_{1}(c)\right]-c_{-}[h]}{\left[c+q_{1}(c)\right]-c_{+}[h]}
$$

where $[c]=c_{+}-c_{-}$and $[h]=h\left(c_{+}\right)-h\left(c_{-}\right)=h_{+}-h_{-}$, and we get (34) thanks to the following computations:

$$
\begin{aligned}
{\left[c+q_{1}(c)\right]-c_{-}[h] } & =\left[c+q_{1}(c)\right]-c_{-} \frac{[u]\left[c+q_{1}(c)\right]}{[u c]}=\frac{\left[c+q_{1}(c)\right]}{[u c]}\left([u c]-c_{-}[u]\right) \\
& =\frac{[c] u_{+}}{[u c]}\left[c+q_{1}(c)\right], \\
{\left[c+q_{1}(c)\right]-c_{+}[h] } & =\left[c+q_{1}(c)\right]-c_{+} \frac{[u]\left[c+q_{1}(c)\right]}{[u c]}=\frac{\left[c+q_{1}(c)\right]}{[u c]}\left([u c]-c_{+}[u]\right) \\
& =\frac{[c] u_{-}}{[u c]}\left[c+q_{1}(c)\right] .
\end{aligned}
$$

Rewriting (34) we get

$$
\begin{aligned}
\frac{u_{-}}{u_{+}} & =\frac{\left[c+q_{1}(c)\right]-c_{+}[h]}{\left[c+q_{1}(c)\right]-c_{-}[h]}=\frac{\left[q_{1}\right]+[c]-c_{+}[h]}{\left[q_{1}\right]+[c]-c_{-}[h]}=\frac{\left[q_{1}\right]+[c]+c_{+}\left(h_{-}-h_{+}\right)}{\left[q_{1}\right]+[c]+c_{-}\left(h_{-}-h_{+}\right)} \\
& =\frac{\left[q_{1}\right]-c_{+} h_{+}+[c]+h_{-} c_{+}}{\left[q_{1}\right]+c_{-} h_{-}+[c]-h_{+} c_{-}}=\frac{[f]+[c]+h_{-}[c]}{[f]+[c]+h_{+}[c]}=\frac{\alpha+h_{-}}{\alpha+h_{+}},
\end{aligned}
$$

which concludes the proof.

We need to know the sign of $\alpha+h_{ \pm}$before studying the sign of $\frac{\partial S}{\partial c_{ \pm}}$.

Lemma 5.2. If $h^{\prime} \leq 0$ and $c_{+}<c<c_{-}$then $\alpha+h\left(c_{+}\right) \geq \alpha+h(c) \geq \alpha+h\left(c_{-}\right)>0$. 
Proof. Since $h^{\prime} \leq 0$ and $c_{+}<c_{-}$, we have $h\left(c_{+}\right) \geq h\left(c_{-}\right)$and this is enough to show that $\frac{[f]}{[c]}+1+h\left(c_{-}\right)>0$. This inequality is equivalent to $[f]+[c]+[c] h\left(c_{-}\right)<0$ because $[c]=c_{+}-c_{-}<0$. Since $f(c)=q_{1}(c)-c h(c)$ the inequality is equivalent to $\left[q_{1}\right]+[c]<c_{+}[h]$. We know that $q_{1}^{\prime} \geq 0, c_{+}<c_{-}, h^{\prime} \leq 0$ such that $\left[q_{1}\right] \leq 0,[c]<$ $0,[h] \geq 0$ and thus $\left[q_{1}\right]+[c]<0<c_{+}[h]$. 0 .

LEMma 5.3. If $h^{\prime} \leq 0$, if $f$ is convex and if $c_{+}<c_{-}$then we have $\frac{\partial S}{\partial c_{+}}\left(c_{+}, c_{-}\right) \leq$

Proof. Thanks to Lemma 5.1 , we have $S\left(c_{+}, c_{-}\right)=[L]=\ln \left(u_{+}\right)-$ $\ln \left(u_{-}\right)=\ln \left(\frac{u_{+}}{u_{-}}\right)$and $\frac{\partial}{\partial c_{+}} \frac{u_{+}}{u_{-}}=\frac{\partial}{\partial c_{+}} \frac{\alpha+h_{+}}{\alpha+h_{-}}$. A calculus gives $\frac{\partial}{\partial c_{+}} \frac{\alpha+h_{+}}{\alpha+h_{-}}=$ $\frac{1}{\left(\alpha+h_{-}\right)^{2}}\left(-\frac{\partial \alpha}{\partial c_{+}}[h]+h^{\prime}\left(c_{+}\right)\left(\alpha+h_{-}\right)\right)$. Now $\frac{\partial \alpha}{\partial c_{+}} \geq 0$ because $f$ is convex, next $[h] \geq 0$ since $h^{\prime} \leq 0$ and $c_{+}<c_{-}$. Lastly $\alpha+h_{-}>0$ from Lemma 5.2 and we get $\frac{\partial S}{\partial c_{+}}\left(c_{+}, c_{-}\right) \leq 0$.

The following result concerns the case with an inert gas:

LEMmA 5.4. If $q_{1}=0$ and $q_{2}^{\prime \prime} \leq 0$ then $\frac{\partial S}{\partial c_{-}} \geq 0$ for $c_{-}>c_{+}$.

Proof. If $q_{1}=0$ then $f(c)=-c h(c), h(c)=q_{2}(c)$ then $h^{\prime}(c)=q_{2}^{\prime}(c) \leq 0$. By a direct computation and thanks to Lemma 5.1 , we have

$$
\frac{u_{+}}{u_{-}}=\frac{[c]-c_{-}[h]}{[c]-c_{+}[h]}=\frac{[c]-c_{+}[h]+[c][h]}{[c]-c_{+}[h]}=1+\frac{1}{\frac{1}{[h]}-\frac{c_{+}}{[c]}} .
$$

But as $\frac{\partial}{\partial c_{-}} \frac{1}{[h]}<0$ and $-\frac{c_{+}}{[c]}$ decreases, $\frac{u_{+}}{u_{-}}$increases with respect to $c_{-}$. $\square$

In the case of two active components we have the following result:

Lemma 5.5. If $q_{1}^{\prime \prime} \leq 0 \leq q_{2}^{\prime \prime}$ and if $f$ is convex then $\frac{\partial S}{\partial c_{-}}\left(c_{+}, c_{-}\right) \geq 0$.

Proof. Let $c$ be between $c_{+}$and $c_{-}$. From Lemma 5.2 we get:

$$
u(c)=\frac{f\left(c_{+}\right)-f(c)}{c_{+}-c}+1+h\left(c_{+}\right)>0, \quad v(c)=\frac{f\left(c_{+}\right)-f(c)}{c_{+}-c}+1+h(c)>0 .
$$

We rewrite $S$ using the functions $u, v$. With Lemma 5.1 we immediately get:

$$
\begin{aligned}
S\left(c_{+}, c_{-}\right) & =\ln \left(\frac{[f] /[c]+1+h_{+}}{[f] /[c]+1+h_{-}}\right) \\
& =\ln \left(\frac{u\left(c_{-}\right)}{v\left(c_{-}\right)}\right) .
\end{aligned}
$$

The function $f$ is convex, so $u$ is increasing. From equality $f(c)=q_{1}(c)-\operatorname{ch}(c)$ we have

$$
\begin{aligned}
(v(c)-1)\left(c_{+}-c\right) & =q_{1}\left(c_{+}\right)-c_{+} h\left(c_{+}\right)-q_{1}(c)+\operatorname{ch}(c)+h(c)\left(c_{+}-c\right) \\
& =q_{1}\left(c_{+}\right)-q_{1}(c)-c_{+}\left(h\left(c_{+}\right)-h(c)\right) .
\end{aligned}
$$


Recall that $h(c)=q_{1}(c)+q_{2}(c)$, so we have:

$$
\begin{aligned}
(v(c)-1)\left(c_{+}-c\right) & =q_{1}\left(c_{+}\right)-q_{1}(c)-c_{+}\left(q_{1}\left(c_{+}\right)+q_{2}\left(c_{+}\right)-q_{1}(c)-q_{2}(c)\right) \\
& =\left(1-c_{+}\right)\left(q_{1}\left(c_{+}\right)-q_{1}(c)\right)-c_{+}\left(q_{2}\left(c_{+}\right)-q_{2}(c)\right) .
\end{aligned}
$$

Finally, $v(c)-1=\left(1-c_{+}\right) \frac{q_{1}\left(c_{+}\right)-q_{1}(c)}{c_{+}-c}-c_{+} \frac{q_{2}\left(c_{+}\right)-q_{2}(c)}{c_{+}-c}$ with $0 \leq c_{+} \leq 1$. Now, $q_{1}$ is concave and $q_{2}$ is convex, so $v$ is decreasing. Finally, $\frac{u}{v}$ is increasing and $\frac{\partial S}{\partial c_{-}} \geq 0$.

6. Interactions estimates. In this section we study the evolution of the total variation of $L=\ln (u)$, noted $T V L$, through waves interactions. It is a key point to obtain some $B V$ bounds and a special structure for the velocity.

Let us note $\left(c_{0}, L_{0}\right),\left(c_{1}, L_{1}\right),\left(c_{2}, L_{2}\right)$, three constant states such that:

- the Riemann problem with $\left(c_{0}, L_{0}\right)$ for the left state and $\left(c_{1}, L_{1}\right)$ for the right state is solved by a simple wave $\mathcal{W}_{1}$,

- the Riemann problem with $\left(c_{1}, L_{1}\right)$ for the left state and $\left(c_{2}, L_{2}\right)$ for the right state is solved by a simple wave $\mathcal{W}_{2}$,

- $\mathcal{W}_{1}$ and $\mathcal{W}_{2}$ interact.

Just after the interaction we have two outgoing waves $\mathcal{W}_{1}^{*}, \mathcal{W}_{2}^{*}$, and the intermediary constant state $\left(c_{1}^{*}, L_{1}^{*}\right)$. We note by $T V L$ the total variation of $\ln u$ just before interaction:

$$
T V L=\left|L_{0}-L_{1}\right|+\left|L_{1}-L_{2}\right| .
$$

We note by $T V L^{*}$ the total variation of $\ln u$ just after the interaction:

$$
T V L^{*}=\left|L_{0}-L_{1}^{*}\right|+\left|L_{1}^{*}-L_{2}\right| .
$$

We use a similar notation for the concentration.

Let us note by $\alpha_{-}$the negative part of $\alpha: \alpha_{-}=\max (0,-\alpha)=-\min (0, \alpha) \geq 0$. We have the following key estimates:

Theorem 6.1 (Variation of $T V \ln u$ and $T V c$ through two waves interaction). Assume (30). Then there exists a constant $\Gamma \geq 0$ such that:

$$
\begin{aligned}
T V L^{*} & \leq T V L+\Gamma\left|c_{0}-c_{1}\right|\left|c_{1}-c_{2}\right|, \\
T V c^{*} & \leq T V c .
\end{aligned}
$$

Furthermore, if (31) is also satisfied then:

$$
T V L^{*} \leq T V L+\Gamma\left(c_{1}-c_{0}\right)_{-} \times\left(c_{2}-c_{1}\right)_{-},
$$

in addition, if $S$, from (32), satisfies the following triangular inequality:

$$
\left(c_{2}, c_{0}\right) \leq S\left(c_{2}, c_{1}\right)+S\left(c_{1}, c_{0}\right)
$$

when $c_{0}>c_{1}>c_{2}$, then

$$
T V L^{*} \leq T V L .
$$

Inequality (36) means that the total variation of $c$ does not increase and Inequality (37) means that the total variation of $\ln u$ does not increase after a wave interaction 
except when two shocks interact. If (30), (31) and (38) are satisfied then (39) means (35) holds with $\Gamma=0$. In this last case the increase of $T V \ln u$ is quadratic with respect to the concentration variation.

Such estimates are only valid when $f$ has no inflexion point. Conversely, $\lambda$-wave curves are only Lipschitz and we lose the quadratic control for the total variation of $L$.

Proof of Inequality (36). The decay of the total variation of the concentration is straightforward since $c$ is constant through a contact discontinuity, i.e. $c_{1}^{*}=c_{0}$ : $T V c^{*}=\left|c_{2}-c_{1}^{*}\right|+\left|c_{1}^{*}-c_{0}\right|=\left|c_{2}-c_{0}\right| \leq\left|c_{0}-c_{1}\right|+\left|c_{1}-c_{2}\right|=T V c$.

Proof of Inequality (35). This proof is much more complicated. We only assume (30). The proof is a consequence of the following lemmas.

Lemma 6.1. If a $\lambda$-wave interacts with a contact discontinuity then we have $T V L^{*}=T V L$.

Proof. It is the simplest case. We have $c_{1}=c_{2}$ from the contact discontinuity, so, with $T$ defined in (32), $L_{1}-L_{0}=T\left(c_{1}, c_{0}\right)=T\left(c_{2}, c_{0}\right)$ and, since $c_{1}^{*}=c_{0}$, we have $L_{2}-L_{1}^{*}=T\left(c_{2}, c_{1}^{*}\right)=T\left(c_{2}, c_{0}\right)$. Then

$$
L_{2}-L_{1}^{*}=L_{1}-L_{0},
$$

which implies $L_{2}-L_{1}=L_{1}^{*}-L_{0}$ and $T V L^{*}=T V L$.

Lemma 6.2. There exists a constant $\Gamma>0$ such that, for all $c_{0}, c_{1}, c_{2} \in[0,1]$ :

$$
\left|T\left(c_{2}, c_{0}\right)-T\left(c_{2}, c_{1}\right)-T\left(c_{1}, c_{0}\right)\right||\leq \Gamma| c_{2}-c_{1}|| c_{1}-c_{0} \mid .
$$

Proof. We define $R$ by $R(\alpha, \beta)=T\left(c_{2}, c_{0}\right)-T\left(c_{2}, c_{1}\right)-T\left(c_{1}, c_{0}\right)$. We have to prove that $R(\alpha, \beta)=\mathcal{O}(\alpha \beta)$, where $\alpha=c_{1}-c_{0}, \beta=c_{1}-c_{2}$. We note $c=c_{2}, b=c_{1}, a=c_{0}$. We have $T \in \mathcal{C}^{3}([0,1], \mathbb{R})$ since $\lambda$ is genuinely nonlinear. Notice that $T(b, b)=0$. We now apply the Taylor's formula:

$$
\begin{aligned}
T(c, a)= & T(b-\beta, b+\alpha)=T(b, b)-\beta \partial_{1} T(b, b)+\alpha \partial_{2} T(b, b) \\
& +\int_{0}^{1}(1-t)\left(\beta^{2} \partial_{1}^{2} S+\alpha^{2} \partial_{2}^{2} T-2 \alpha \beta \partial_{12}^{2} T\right)(b-t \beta, b+t \alpha) d t, \\
T(b, a)= & T(b, b+\alpha)=T(b, b)+\alpha \partial_{2} T(b, b)+\int_{0}^{1}(1-t) \alpha^{2} \partial_{2}^{2} T(b, b+t \alpha) d t, \\
T(c, b)= & T(b-\beta, b)=T(b, b)-\beta \partial_{1} T(b, b)+\int_{0}^{1}(1-t) \beta^{2} \partial_{1}^{2} T(b-t \beta, b) d t, \\
R(\alpha, \beta)= & T(c, a)-T(c, b)-T(b, a) \\
= & -T(b, b)+\int_{0}^{1}(1-t)\left(\beta^{2}\left(\partial_{1}^{2} T(b-t \beta, b+t \alpha)-\partial_{1}^{2} T(b-t \beta, b)\right)+\right. \\
& \left.\alpha^{2}\left(\partial_{2}^{2} T(b-t \beta, b+t \alpha)-\partial_{2}^{2} T(b, b+t \alpha)\right)-2 \alpha \beta \partial_{1} \partial_{2} T(b-t \beta, b+t \alpha)\right) d t .
\end{aligned}
$$

Since

$$
\begin{aligned}
\partial_{1}^{2} T(b-t \beta, b+t \alpha)-\partial_{1}^{2} T(b-t \beta, b) & =\mathcal{O}(t \alpha)=\mathcal{O}(\alpha), \\
\partial_{2}^{2} T(b-t \beta, b+t \alpha)-\partial_{2}^{2} T(b, b+t \alpha) & =\mathcal{O}(t \beta)=\mathcal{O}(\beta), \\
\partial_{1} \partial_{2} T(b-t \beta, b+t \alpha) & =\mathcal{O}(1)
\end{aligned}
$$


we conclude that $R(\alpha, \beta)=\mathcal{O}\left(\beta^{2} \alpha+\alpha^{2} \beta+\alpha \beta\right)=\mathcal{O}(\alpha \beta)$.

To conclude the proof of Inequality (35) it suffices to use the next lemma.

Lemma 6.3. If two $\lambda$-waves interact then we have

$$
T V L^{*} \leq T V L+\Gamma\left|c_{2}-c_{1}\right|\left|c_{1}-c_{0}\right| .
$$

Proof. By definition of $T V L$ and $T V L^{*}$ it suffices to prove that

$$
L_{1}^{*}=L_{0}+\mathcal{O}\left(\left|c_{2}-c_{1}\right|\left|c_{1}-c_{0}\right|\right),
$$

since $T V L^{*}=\left|L_{2}-L_{1}^{*}\right|+\left|L_{1}^{*}-L_{0}\right| \leq\left|L_{2}-L_{0}\right|+2\left|L_{1}^{*}-L_{0}\right| \leq T V L+2\left|L_{1}^{*}-L_{0}\right|$. Indeed, we have: $L_{1}-L_{0}=T\left(c_{1}, c_{0}\right), L_{2}-L_{1}=T\left(c_{2}, c_{1}\right), L_{2}-L_{1}^{*}=T\left(c_{2}, c_{1}^{*}\right)=$ $T\left(c_{2}, c_{0}\right)$. Next: $L_{2}-L_{0}=T\left(c_{2}, c_{1}\right)+T\left(c_{1}, c_{0}\right)$ and then

$$
L_{1}^{*}-L_{0}=T\left(c_{2}, c_{1}\right)+T\left(c_{1}, c_{0}\right)-T\left(c_{2}, c_{0}\right),
$$

which allows us to conclude the proof of Lemma 6.3 with Lemma 6.2.

The proof of Inequality (35) is now complete.

Proof of Inequalities (37), (39). We assume again (31) and also (33) to fix the signs. There are more cases to study:

- first, we have already studied in Lemma 6.1 the interaction of a shock wave or a rarefaction wave ( $\lambda$-wave) with a contact discontinuity (1-wave): the contact discontinuity is "transparent" since $T V L^{*}=T V L$ and the concentration variation is also invariant.

- second, we study the interaction of a shock wave with a rarefaction wave ( $\lambda$-waves with different types): see Lemmas $6.4,6.5,6.6$ and 6.7. We get $T V L^{*}<T V L$ and the concentration variation decreases. It is the only case where $T V L$ and $T V c$ decrease.

- finally, we study the interaction of two shock waves. In this situation $T V L^{*} \geq$ $T V L$ and $T V c$ is invariant.

Furthermore, if $S$ satisfies some "triangular inequality", we get $T V L^{*}=$ $T V L$.

In order to simplify the notations we note by $\mathrm{D}$ a contact discontinuity, $\mathrm{R}$ a rarefaction wave and S a shock wave. " $\mathrm{RD} \rightarrow \mathrm{DR}$ " means that a rarefaction wave coming from the left interacts with a contact discontinuity and produces a new left wave, namely a contact discontinuity, and a new right wave, namely a rarefaction.

Since a contact discontinuity has a null speed and a $\lambda$-wave has a positive speed, the only cases for $\mathcal{W}_{1}, \mathcal{W}_{2}$ are: RD, SD, RS, SR and SS.

For the resulting waves $\mathcal{W}_{1}^{*}, \mathcal{W}_{2}^{*}$, there are 7 cases.

The first two cases $\mathrm{RD} \rightarrow \mathrm{DR}$ and $\mathrm{SD} \rightarrow \mathrm{DS}$ have already been studied in Lemma 6.1 .

Lemma 6.4. In the case $R S \rightarrow D R, T V L$ decreases i.e. $T V L^{*}<T V L$.

Proof. In the beginning, we have a rarefaction, so $c_{0}<c_{1}, L_{0}>L_{1}$, then we have a shock, so $c_{2}<c_{1}, L_{2}>L_{1}$. After the interaction, we have a contact discontinuity, so $c_{0}=c_{1}^{*}$, then a rarefaction, so $c_{1}^{*}<c_{2}, L_{1}^{*}>L_{2}$. Finally, we have $c_{0}=c_{1}^{*}<c_{2}<c_{1}$ so $g\left(c_{0}\right)=g\left(c_{1}^{*}\right) \leq g\left(c_{2}\right) \leq g\left(c_{1}\right)$. We can write

$$
\begin{aligned}
T V L & =\left|L_{0}-L_{1}\right|+\left|L_{1}-L_{2}\right|=L_{0}-L_{1}+L_{2}-L_{1}, \\
T V L^{*} & =\left|L_{0}-L_{1}^{*}\right|+\left|L_{2}-L_{1}^{*}\right|=\left|L_{0}-L_{1}^{*}\right|+L_{1}^{*}-L_{2} .
\end{aligned}
$$

There are two cases: 
- the simplest is $L_{0}>L_{1}^{*}$, then $T V L^{*}=L_{0}-L_{1}^{*}+L_{1}^{*}-L_{2}=L_{0}-L_{2}<$ $L_{0}-L_{1}<T V L$

- the second case is $L_{0}<L_{1}^{*}$. Let us define $\tilde{L}_{2}$ by

$$
L_{0}-\tilde{L}_{2}=L_{1}^{*}-L_{2},
$$

then $L_{0}-\tilde{L}_{2}=L_{1}^{*}-L_{2}=g\left(c_{2}\right)-g\left(c_{1}^{*}\right)=g\left(c_{2}\right)-g\left(c_{0}\right) \leq g\left(c_{1}\right)-g\left(c_{0}\right)=$ $L_{0}-L_{1}$ because $[L]=-[g]$ for a rarefaction and $c_{1}^{*}=c_{0}$. Since shock curves are decreasing, we know that $\tilde{L}_{2}>L_{1}$, so $T V L^{*}=L_{1}^{*}-L_{0}+L_{1}^{*}-L_{2}=$ $L_{2}-\tilde{L}_{2}+L_{0}-\tilde{L}_{2}<L_{2}-L_{1}+L_{0}-L_{1}=T V L$.

Lemma 6.5. In the case $R S \rightarrow D S$ we get $T V L^{*} \leq T V L$.

Proof. This case needs the assumption $\frac{\partial S}{\partial c_{-}} \geq 0$. In the beginning, we have a rarefaction: $c_{1}>c_{0}$ and $L_{1}<L_{0}$ with a shock: $c_{2}<c_{1}$ and $L_{2}>L_{1}$. The state $\left(c_{2}, L_{2}\right)$ is connected with a shock $\left(c_{1}^{*}, L_{1}^{*}\right): c_{2}<c_{1}^{*}$ and $L_{1}^{*}<L_{2}$. The state $\left(c_{0}, L_{0}\right)$ is connected with a contact discontinuity $\left(c_{1}^{*}, L_{1}^{*}\right): c_{0}=c_{1}^{*}$. Finally, we have $c_{2}<c_{0}=c_{1}^{*}<c_{1}$. Then $T V L=\left|L_{0}-L_{1}\right|+\left|L_{1}-L_{2}\right|=L_{0}-L_{1}+L_{2}-L_{1}$ and $T V L^{*}=\left|L_{0}-L_{1}^{*}\right|+\left|L_{2}-L_{1}^{*}\right|=L_{2}-L_{1}^{*}+\left|L_{1}^{*}-L_{0}\right|$. But, with the assumption, $\frac{\partial S}{\partial c_{-}} \geq 0, S\left(c_{2}, c_{0}\right)=S\left(c_{2}, c_{1}^{*}\right)=L_{2}-L_{1}^{*}<S\left(c_{2}, c_{1}\right)=L_{2}-L_{1}$ then $L_{1}^{*}>L_{1}$.

There are two cases:

- if $L_{0}>L_{1}^{*}$ then $T V L^{*}=L_{0}-L_{1}^{*}+L_{2}-L_{1}^{*}<L_{0}-L_{1}+L_{2}-L_{1}=T V L$,

- otherwise $L_{0}<L_{1}^{*}$ then $T V L^{*}=-L_{0}+L_{1}^{*}+L_{2}-L_{1}^{*}=L_{2}-L_{0}<L_{2}-L_{1}<$ TVL.

Lemma 6.6. In the case $S R \rightarrow D R$ we have $T V L^{*} \leq T V L$.

Proof. In the beginning, we have a shock that interacts with a rarefaction so $c_{1}<c_{0}, L_{1}>L_{0}$ and $c_{2}>c_{1}, L_{1}>L_{2}$.

After the interaction, we have a contact discontinuity so $c_{0}=c_{1}^{*}$ then a rarefaction so $c_{2}>c_{1}^{*}$ and $L_{1}^{*}>L_{2}$. Finally, we have $c_{1}<c_{0}=c_{1}^{*}<c_{2}$. Since $g^{\prime} \geq 0$, we have $g\left(c_{1}\right) \leq g\left(c_{0}\right) \leq g\left(c_{2}\right)$.

For a rarefaction $[L]=-[g]$ so $L_{2}-L_{1}^{*}=g\left(c_{1}^{*}\right)-g\left(c_{2}\right)=g\left(c_{0}\right)-g\left(c_{2}\right)$ because $c_{1}^{*}=c_{0}$, $L_{2}-L_{1}=g\left(c_{1}\right)-g\left(c_{2}\right) \leq g\left(c_{0}\right)-g\left(c_{2}\right)$ because $c_{1}<c_{0}$ and $g^{\prime} \geq 0$.

So we have: $L_{2}-L_{1} \leq g\left(c_{1}^{*}\right)-g\left(c_{2}\right)=L_{2}-L_{1}^{*}$ and

$T V L=\left|L_{1}-L_{0}\right|+\left|L_{2}-L_{1}\right|=L_{1}-L_{0}+L_{1}-L_{2} \geq L_{1}-L_{2}$,

$T V L^{*}=\left|L_{1}^{*}-L_{0}\right|+\left|L_{2}-L_{1}^{*}\right|=\left|L_{1}^{*}-L_{0}\right|+L_{1}^{*}-L_{2}$.

There are two cases:

- the first is $L_{1}^{*}>L_{0}$ then $T V L^{*}=L_{1}^{*}-L_{0}-L_{2}+L_{1}^{*}=2 L_{1}^{*}-L_{0}-L_{2}=$ $-\left(L_{2}-L_{1}^{*}\right)+L_{1}^{*}-L_{0}<-\left(L_{2}-L_{1}\right)+L_{1}^{*}-L_{2}+L_{2}-L_{0}<-L_{2}+L_{1}-L_{2}+$ $L_{1}+L_{2}-L_{0}=2 L_{1}-L_{2}-L_{0}=T V L$,

- the second case is $L_{1}^{*}<L_{0}$ then $T V L^{*}=-L_{1}^{*}+L_{0}-L_{2}+L_{1}^{*}=L_{0}-L_{2} \leq$ $L_{1}-L_{2} \leq T V L$.

Lemma 6.7. In the case $S R \rightarrow D S, T V L$ decreases i.e. $T V L^{*} \leq T V L$.

This situation is illustrated in Fig. 3.

Proof. It is the most difficult case. In the beginning, we have a shock so $c_{1}<c_{0}$ and $L_{1}>L_{0}$. The shock interacts with a rarefaction so $c_{2}>c_{1}$ and $L_{2}<L_{1}$.

We then have $T V L=\left|L_{1}-L_{0}\right|+\left|L_{2}-L_{1}\right|=L_{1}-L_{0}+L_{1}-L_{2}$. 
The state $\left(c_{2}, L_{2}\right)$ is connected to $\left(c_{1}^{*}, L_{1}^{*}\right)$ by a shock so $c_{2}<c_{1}^{*}$ and $L_{1}^{*}<L_{2}$.

The state $\left(c_{0}, L_{0}\right)$ is connected to $\left(c_{1}^{*}, L_{1}^{*}\right)$ by a contact discontinuity so $c_{0}=c_{1}^{*}$.

Finally, we have $c_{1}<c_{2}<c_{1}^{*}=c_{0}, S\left(c_{1}, c_{0}\right)=S_{10}>S\left(c_{2}, c_{0}\right)=S_{20}=S\left(c_{2}, c_{1}^{*}\right)=$ $L_{2}-L_{1}^{*}, L_{1}-L_{0}=S_{10}>S_{20}=L_{2}-L_{1}^{*}$, because $\frac{\partial S}{\partial c_{+}}<0$.

There are two cases:

- if $L_{0}<L_{1}^{*}$ (see Fig. 4, left) then $L_{2}<L_{1}$ and $T V L^{*}=\left|L_{1}^{*}-L_{0}\right|+\left|L_{2}-L_{1}^{*}\right|=L_{1}^{*}-L_{0}+L_{2}-L_{1}^{*}=L_{2}-L_{0}<L_{1}-L_{0}<T V L$,

- if $L_{1}^{*}<L_{0}$ (see Fig. 4, right) then we define $\tilde{L}_{2}$ by $\tilde{L}_{2}-L_{0}=S_{20}=L_{2}-L_{1}^{*}<$ $S_{10}=L_{1}-L_{0}$ and $T V L^{*}=\left|L_{1}^{*}-L_{0}\right|+\left|L_{2}-L_{1}^{*}\right|=L_{0}-L_{1}^{*}+L_{2}-L_{1}^{*}=$ $\tilde{L}_{2}-L_{2}+S_{20}<L_{1}-L_{0}+L_{1}-L_{0}=T V L$.

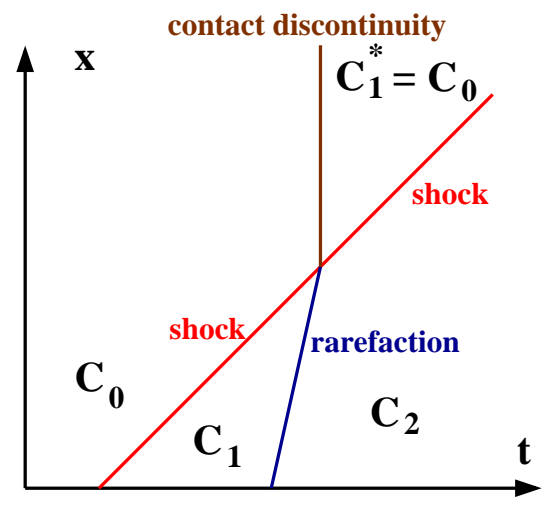

FIG. 3. Case $S R \rightarrow D S$.
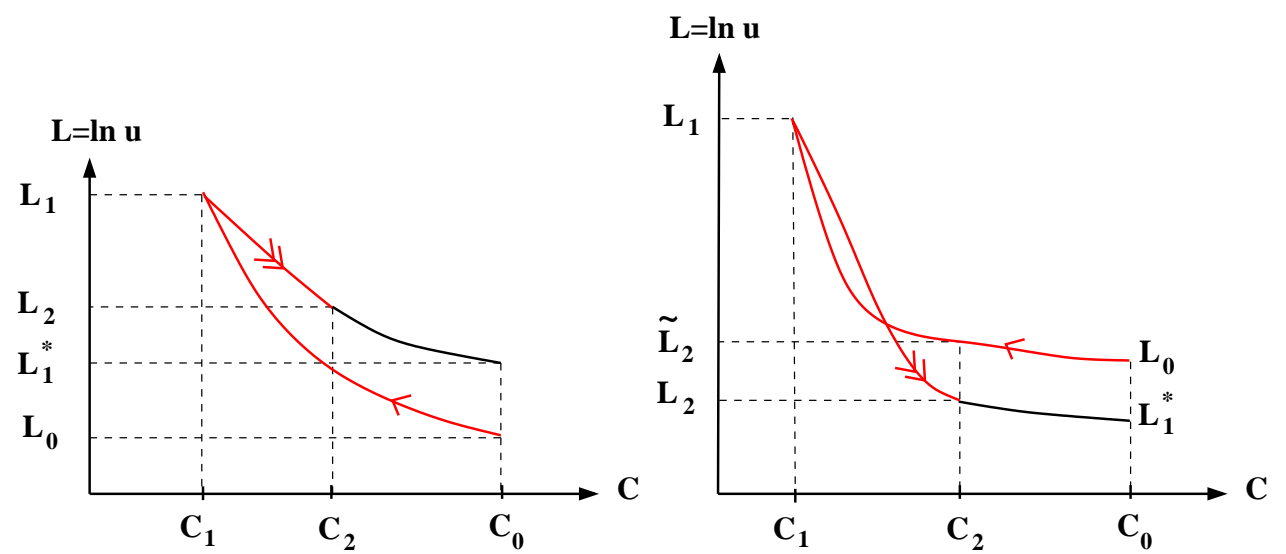

FIG. 4. SR $\rightarrow$ DS: first case, left, and second case, right. 
The following case is the only one where $T V L$ increases, except if $S$ satisfies a "triangular inequality".

Lemma 6.8. In the case $S S \rightarrow D S$ we have

$T V L^{*}=T V L+2 \max \left(S_{20}-S_{21}-S_{10}, 0\right)=T V L+2 \max \left(L_{0}-L_{1}^{*}, 0\right) \geq T V L$.

Proof. In the beginning, we have a shock: $c_{1}<c_{0}$ and $L_{1}>L_{0}$. It interacts with an another shock: $c_{2}<c_{1}$ and $L_{2}>L_{1}$.

The state $\left(c_{2}, L_{2}\right)$ is connected to $\left(c_{1}^{*}, L_{1}^{*}\right)$ by a shock so $c_{2}<c_{1}^{*}$ and $L_{1}^{*}<L_{2}$.

The state $\left(c_{0}, L_{0}\right)$ is connected with $\left(c_{1}^{*}, L_{1}^{*}\right)$ by a contact discontinuity so $c_{0}=c_{1}^{*}$.

Finally, we have $c_{2}<c_{1}<c_{0}=c_{1}^{*}$ and $L_{0}<L_{1}<L_{2}$.

With $L_{2}-L_{1}=S_{21}>0, L_{1}-L_{0}=S_{10}>0, L_{2}-L_{1}^{*}=S_{20}>0$, we have:

$T V L=\left|L_{2}-L_{1}\right|+\left|L_{1}-L_{0}\right|=L_{2}-L_{1}+L_{1}-L_{0}=S_{21}+S_{10}$,

$T V L^{*}=\left|L_{2}-L_{1}^{*}\right|+\left|L_{1}^{*}-L_{0}\right|=\left|S_{20}\right|+\left|L_{1}^{*}-L_{2}+L_{2}-L_{0}\right|=S_{20}+\mid$ $-S_{20}+L_{2}-L_{0} \mid$.

There are two cases to study:

- if $-S_{20}+L_{2}-L_{0} \geq 0$ i.e. $S_{20}=L_{2}-L_{1}^{*} \leq S_{21}+S_{10}=L_{2}-L_{0}$ i.e. $L_{0}<L_{1}^{*}$ then $T V L^{*}=S_{20}-S_{20}+L_{2}-L_{0}=L_{2}-L_{0}=T V L$,

- otherwise $L_{1}^{*}<L_{0}$ and we have

$$
\begin{aligned}
T V L^{*} & =S_{20}+S_{20}-L_{2}+L_{0}=2 S_{20}-2 L_{2}+2 L_{0}+L_{2}-L_{0} \\
& =2\left(S_{20}-\left(L_{2}-L_{0}\right)\right)+T V L,=2\left(S_{20}-S_{21}-S_{10}\right)+T V L \\
& =2\left(L_{0}-L_{1}^{*}\right)+T V L,
\end{aligned}
$$

which concludes the proof of Lemma 6.8.

The proof of Theorem 6.1 is now complete.

7. $B V$ estimates with respect to time for the velocity. In System (1)(2)-(3), there is no partial derivative with respect to $t$ for $u$. Nevertheless, the hyperbolicity of this system ( with $x$ as the evolution variable) suggests that a $B V$ regularity of the "initial" data $u_{b}$ for $x=0$ is propagated. Furthermore, in the case with smooth concentration, the Riemann invariant $u G(c)$ suggests that when $\ln u_{b}$ is only in $L^{\infty}(0, T)$, we can hope $u(t, x) / u_{b}(t)$ to be still $B V$ in time for almost all $x$. We prove that this $B V$ structure of the velocity is still valid with a convex assumption, using a Front Tracking Algorithm (FTA). We conjecture that this structure is still valid for the general case without convex assumption, i.e. with a piecewise genuinely nonlinear eigenvalue $\lambda=H(c) / u$. But, in this last case, the FTA becomes very complicated (see Dafermos' comments in [21]).

7.1. The case $\ln u_{b} \in B V(0, T)$. We first precise the notations used in the next theorem. We define the function $c_{I}$ on $(0, T)$ by

$$
c_{I}(s)=\left\{\begin{array}{cl}
c_{0}(s) & \text { if } 0<s<X \\
c_{b}(-s) & \text { if } 0<-s<T
\end{array},\right.
$$

and we set $T V c_{I}=T V c_{I}[-T, X]$.

There exists a positive constant $\gamma$ such that if $\left(c_{-}, L_{-}\right)$is connected to $\left(c_{+}, L_{+}\right)$by a $\lambda$-wave then $\left|L_{+}-L_{-}\right| \leq \gamma\left|c_{+}-c_{-}\right|$. That is a simple consequence of (32). Indeed, it is already proven in [9], Lemma 3.1, with an inert gas, or in [10], Lemma 4.1, for two active gases.

The constant $\Gamma$ comes from Theorem 6.1 . 
TheOREM 7.1 (Propagation of BV regularity with respect to time for the velocity). Assume (30). If $\ln u_{b} \in B V(0, T)$, if $c_{0}, c_{b} \in B V$ and if $(u, c)$ is a weak entropy solution of System (1)-(2)-(3), coming from the Front Tracking Algorithm, then $c \in B V((0, T) \times(0, X))$ and $u \in L^{\infty}((0, T), B V(0, X)) \cap L^{\infty}((0, X), B V(0, T))$. More precisely:

$$
\begin{aligned}
\max \left(\sup _{0<t<T} T V_{x} c(t, .)[0, X],\right. & \left.\sup _{0<x<X} T V_{t} c(., x)[0, T]\right) \leq T V c_{I}, \\
& \sup _{0<t<T} T V_{x} \ln u(t, .)[0, X] \leq T V \ln u_{b}+\gamma T V c_{I}, \\
& \sup _{0<x<X} T V_{t} \ln u(., x)[0, T] \leq T V \ln u_{b}+2 \gamma T V c_{I}+\frac{\Gamma}{2}\left(T V c_{I}\right)^{2} .
\end{aligned}
$$

Furthermore, if (30), (31) and (38) are satisfied then $\Gamma=0$ in the last inequality.

Compared to $[9,10]$, the new result is that $u(t, x)$ is BV with respect to time if $u_{b}$ is in $B V(0, T)$ i.e. the last inequality of Theorem 7.1. This is the stratified structure (8) for the velocity with $B V$ concentration. With the Godunov scheme used in $[9,10]$, we did not obtain such time regularity for the velocity. It is the reason why we use the FTA to get more precise estimates. Notice that we consider a local problem (in time and space) for realism reasons ; we could have considered a global one as well, i.e. for $(t, x) \in(0,+\infty)^{2}$.

Proof. The easiest BV estimate on the concentration $c$ after interaction (estimate (36) in Theorem 6.1), which is always valid independently of the velocity $u$, yields to a control of $c$ in $L_{t}^{\infty} B V_{x} \cap L_{x}^{\infty} B V_{x}$ as in [10], since $\lambda$ waves always have a positive speed. From Lemma 4.8 of [10] p.80 (or more simply Lemma 3.1 of [9] p. 557) we get $L_{t, x}^{\infty} \cap L_{t}^{\infty} B V_{x}$ bounds for the velocity $u$. It follows, from a natural adaptation of the estimates and of the compactness argument in the proof of Theorem $5.1 \mathrm{p} 563$. in [9] or Theorem 6.1 p.83 in [10], that there exists a subsequence which converges to a solution of the initial boundary value problem with the prescribed data $c_{0}, c_{b}, u_{b}$ when $\delta$ goes to zero, thanks to the approximate entropy inequality (29). Furthermore, as in $[9,10]$, we recover strong traces at $t=0$ and $x=0$.

Notice that this existence proof is also valid without any $B V$ assumption on the velocity at the boundary: we only need $\ln u_{b}$ in $L^{\infty}(0, T)$.

The $B V$ estimate with respect to time for $\ln u$, i.e. the third estimate in the theorem, is a consequence of the two following lemmas.

Let $(u, c)$ be an entropy solution coming from FTA. For $\delta>0$, representing the distance from the boundary $x=0$ or $t=0$, let us define:

$$
\begin{aligned}
& L(s, \delta)=\left\{\begin{array}{cc}
\ln u(t=|s|, x=\delta) & \text { if }-T<s<0 \\
\ln u(t=\delta, x=s) & \text { if } 0<s<X
\end{array},\right. \\
& T V L(0)=\limsup _{\delta \rightarrow 0} T V L(., \delta)[-T, X] \text {. }
\end{aligned}
$$

For piecewise data, $T V L(0)$ is the total variation of $\ln u$ just before the first interaction.

LEMma 7.1. Before wave-interactions we have $T V L(0) \leq T V \ln u_{b}+2 \gamma T V c_{I}$.

Proof. It suffices to prove this inequality for a piecewise constant approximate solution issued from the FTA. We discretize $[0, T]$ and $[0, X]$ as follows: 
$T=s_{1}>s_{2} \cdots>s_{m}>s_{m+1}=0<s_{m+2}<\cdots<s_{N}=X$.

For $i=1, \cdots, m$ let us define the following piecewise approximations of $c$ and $\ln u$ :

$$
c_{i}=\frac{1}{s_{i}-s_{i+1}} \int_{s_{i+1}}^{s_{i}} c_{b}(t) d t, L_{i}=\frac{1}{s_{i}-s_{i+1}} \int_{s_{i+1}}^{s_{i}} \ln \left(u_{b}(t)\right) d t .
$$

Since $t=0$ is a characteristic boundary, we define only $c_{i}$ for $i=m+1, \cdots, N-1$ by:

$$
c_{i}=\frac{1}{s_{i}-s_{i+1}} \int_{s_{i+1}}^{s_{i}} c_{0}(x) d x .
$$

For $i<m$ we solve the $i^{\text {th }}$ Riemann Problem with left state $\left(c_{i}, L i\right)$ and right state $\left(c_{i+1}, L_{i+1}\right)$ and we note $c_{i}^{*}, L_{i}^{*}$ the intermediate state. Indeed $c_{i}^{*}=c_{i+1}$ since $c$ is constant through a contact discontinuity. From Lemma 3.1 p. 557 of [9] (or Lemma 4.1 p.78-79 of [10] for two active gases) we know that:

$$
\left|L_{i}-L_{i}^{*}\right| \leq \gamma\left|c_{i}-c_{i}^{*}\right|=\gamma\left|c_{i}-c_{i+1}\right| .
$$

We now estimate the total variation of $\ln u$ for the $i^{\text {th }}$ Riemann problem:

$$
\begin{aligned}
\left|L_{i}-L_{i}^{*}\right|+\left|L_{i}^{*}-L_{i+1}\right| & \leq\left|L_{i}-L_{i}^{*}\right|+\left(\left|L_{i}^{*}-L_{i}\right|+\left|L_{i}-L_{i+1}\right|\right) \\
& \leq 2 \gamma\left|c_{i}-c_{i+1}\right|+\left|L_{i}-L_{i+1}\right| .
\end{aligned}
$$

Now, we look at the corner $t=0, x=0$ and $i=m$. There is only one $\lambda$-wave since the boundary is characteristic. With the left state $\left(c_{m}, L_{m}\right)$ and only $\left(c_{m+1}\right)$ for the right state, the resolution of the Riemann problem gives us a new constant value for $\ln u$, namely $L_{m+1}=L_{m}^{*}$. We have again the estimate $\left|L_{m}-L_{m}^{*}\right|=\left|L_{m}-L_{m+1}\right| \leq$ $\gamma\left|c_{m}-c_{m+1}\right|$. So for $i=m+1, m+2, \cdots, N-1$ we define $L_{i}$ solving the characteristic Riemann problems with the estimate:

$$
\left|L_{i}-L_{i+1}\right| \leq \gamma\left|c_{i}-c_{i+1}\right|
$$

Summing up with respect to $i$, we obtain the total variation on $L$ just before the first wave interaction:

$$
\begin{aligned}
T V L & \leq \sum_{i<m}\left(2 \gamma\left|c_{i}-c_{i+1}\right|+\left|L_{i}-L_{i+1}\right|\right)+\sum_{i \geq m} \gamma\left|c_{i}-c_{i+1}\right| \\
& \leq T V \ln u_{b}+2 \gamma T V c_{I} .
\end{aligned}
$$

LEMma 7.2. We have the following estimate: $T V L \leq T V L(0)+\frac{\Gamma}{2}\left(T V c_{I}\right)^{2}$.

Proof. We prove this estimate for any piecewise constant approximation built from the FTA. The same estimate is still true passing to the limit.

First we enumerate, from the left to the right, the absolute values of the concentration jumps for the initial-boundary values :

$$
\alpha_{i}=c_{i}-c_{i-1} \quad i=1, \cdots, N .
$$

Notice that we have $N+1$ constant states for the initial-boundary data: $\left(c_{0}, L_{0}\right), \cdots,\left(c_{N}, L_{N}\right)$. 
From Theorem 6.1 , the increase of the total variation of $\ln u$ is governed by the following inequality $T V L^{*} \leq T V L+\Gamma\left|\alpha_{i-1}\right|\left|\alpha_{i}\right|$ if the wave number $i-1$ interacts with the wave number $i$.

Since $c$ is constant through a contact discontinuity ( $c$ is a 2-Riemann invariant) and the jump of $c$ adds up when two $\lambda$-waves interact, we consider only the interaction between $\lambda$-waves. We measure the strength of the $\lambda$-wave with the jump of $c$ through the wave. We have a positive or a negative sign whether we have a rarefaction or a shock wave.

Let $\left(\alpha_{i}^{k}\right)_{1 \leq i \leq N-k}$ be the strength of the $\lambda$-wave number $i$ (labeled from left to right) after the interaction number $k$. We have $\alpha_{i}^{0}=\alpha_{i}$ and note $j^{k}$ the index such that the interaction number $k$ occurs with the $\lambda$-waves number $j^{k}$ and $j^{k}+1$ where $1<$ $j_{k} \leq N-k$. For $1 \leq i<N-k$, the strengths of $\lambda$-waves after the interaction number $k>0$ are given by:

$$
\alpha_{i}^{k}=\left\{\begin{array}{cc}
\alpha_{i}^{k-1} & \text { if } i<j^{k} \\
\alpha_{i}^{k-1}+\alpha_{i+1}^{k-1} & \text { if } i=j^{k} \\
\alpha_{i+1}^{k-1} & \text { if } i>j^{k}
\end{array},\right.
$$

and the increasing of $T V L$ is less or equal than $\Gamma S^{k}$ where, according to Theorem $6.1, S^{k}$ satisfies:

$$
S^{0}=0, \quad S^{k}=S^{k-1}+\left|\alpha_{i}^{k-1}\right|\left|\alpha_{i+1}^{k-1}\right|
$$

Let us define the integers $l_{i}^{k}$ as follows:

$l_{i}^{0}=i$ and at each interaction

$$
l_{i}^{k}=\left\{\begin{array}{lll}
l_{i}^{k-1} & \text { if } \quad i<j^{k} \\
l_{i+1}^{k-1} & \text { if } \quad i=j^{k}, \ldots, N-k+1 .
\end{array}\right.
$$

Notice that after each interaction with two $\lambda$-waves, there is only one outgoing $\lambda$ wave. Thus, the number of $\lambda$-waves decreases at each interaction, which proves again (see [22]) that the number of interactions is finite and the FTA is well posed.

By induction, we see that: $\alpha_{i}^{k}=\sum_{l_{i}^{k} \leq l<l_{i+1}^{k}} \alpha_{l}$ where $l_{1}^{k}=1<l_{2}^{k}<\cdots<l_{N-k+1}^{k}=$ $N-k+1, l_{i}^{0}=i$ and $l_{i}^{k}$ is non decreasing with respect to $k$. Now, from the definition of $S^{k}$, we can deduce that:

$$
S^{k}=S^{k-1}+\sum_{(i, j) \in J^{k}}\left|\alpha_{i}\right|\left|\alpha_{j}\right|
$$

where $J^{k}=\left\{(i, j) ; l_{j^{k}}^{k-1} \leq i<l_{j^{k}+1}^{k-1} \leq j<l_{j^{k}+2}^{k-1}\right\}$.

Let us check that:

$$
S^{k}=\sum_{(i, j) \in I^{k}}\left|\alpha_{i}\right|\left|\alpha_{j}\right|
$$

where $\emptyset=I^{0} \subset I^{1} \subset \cdots \subset I^{k-1} \subset I^{k} \subset \cdots \subset I=\{(i, j) ; 1 \leq i<j \leq N\}$.

This is true for $k=0$. It is also true for all $k$ if $I^{k-1} \cap J^{k}=\emptyset$ and so $I^{k}=I^{k-1} \cup J^{k}$. The only point to be proved is that $I^{k-1} \cap J^{k}=\emptyset$. The terms $\left|\alpha_{i}\right|\left|\alpha_{j}\right|$ in the last sum of (40) have indexes $i$ and $j$ which appear in two consecutive intervals, i.e. 
$l_{j^{k}}^{k-1} \leq i<l_{j^{k}+1}^{k-1} \leq j<l_{j^{k}+2}^{k-1}$ and after, for $i=j^{k}, l_{i}^{k}=l_{i}^{k-1}$ and $l_{i+1}^{k}=l_{i+2}^{k-1}$. So $i$ and $j$ reside in the same interval and so the terms $\left|\alpha_{i}\right|\left|\alpha_{j}\right|$ cannot appear again in $S^{k+1}, S^{k+2}, \ldots$, since such intervals are not decreasing.

The same is true for all indexes in $I^{k}$. They can appear at most once in $S^{k}$. We then have $I^{k-1} \cap J^{k}=\emptyset$ and (41) is true.

We easily estimate $S^{k}$, which concludes the proof:

$$
S^{k} \leq \sum_{(i, j) \in I}\left|\alpha_{i}\right|\left|\alpha_{j}\right| \leq \frac{1}{2} \sum_{i=1}^{N} \sum_{j=1}^{N}\left|\alpha_{i}\right|\left|\alpha_{j}\right|=\frac{1}{2}\left(\sum_{i=1}^{N}\left|\alpha_{i}\right|\right)^{2} \leq \frac{1}{2}\left(T V c_{I}\right)^{2} .
$$

7.2. The case $\ln u_{b} \in L^{\infty}(0, T)$. For $\ln u_{b} \in L^{\infty}$ and $c_{0}, c_{b} \in B V$ we get a $B V$ structure for the velocity.

THEOREM 7.2 (BV structure for the velocity). We assume (30). If $\ln u_{b} \in$ $L^{\infty}(0, T)$, if $c_{0}, c_{b} \in B V$ and if $(c, u)$ is a weak entropy solution issued from the FTA, then

$$
\max \left(\sup _{0<t<T} T V_{x} c(t, .)[0, X], \sup _{0<x<X} T V_{t} c(., x)[0, T]\right) \leq T V c_{I}
$$

and there exists a function $v$ and constants $\gamma, \Gamma>0$ such that $u(t, x)=u_{b}(t) \times v(t, x)$ with

$$
\begin{gathered}
\ln v \in\left\{L^{\infty}((0, X), B V(0, T)) \cap L^{\infty}((0, T), B V(0, X))\right\} \subset B V((0, T) \times(0, X)), \\
\sup _{0<t<T} T V_{x} \ln v(t, .)[0, X] \leq \gamma T V c_{I}, \\
\sup _{0<x<X} T V_{t} \ln v(., x)[0, T] \leq 2 \gamma T V c_{I}+\frac{\Gamma}{2}\left(T V c_{I}\right)^{2} .
\end{gathered}
$$

Furthermore, if (30), (31) and (38) are satisfied then $\Gamma=0$ in the last inequality.

The new result in this theorem is that $\frac{u(t, x)}{u_{b}(t)}$ is $\mathrm{BV}$ with respect to time, although $u_{b}$ is not assumed to be BV, but just in $L^{\infty}$. The other regularity properties have already been proved in $[9,10]$.

Proof. The first estimates for $c$ are easily obtained as in Theorem 7.1 since the total variation of the concentration does not increase after an interaction. The existence proof of such an entropy solution follows the beginning of the proof of Theorem 7.1, which is a natural adaptation of the existence proof from $[9,10]$ with only $L^{\infty}$ velocity.

We now study the new $B V$ estimates for $v$. We can define $v$ by the relation $u(t, x)=$ $u_{b}(t) v(t, x)$ because $u_{b}>0$. Let $M=\ln v$ and $M_{b}=\ln v(\cdot, x=0)$. The initial total variation of $M$ on $x=0$ is $T V M_{b}=0$ since $v(t, x=0)=1$.

We approach $u_{b}$ with a piecewise constant data (thus in $B V$ ) and we show that the $\mathrm{BV}$ estimate for $M$ is independent of $u_{b}$. Notice the fundamental relation:

$$
[L]=\ln u_{+}-\ln u_{-}=\ln \left(u_{b}(t) v_{+}\right)-\ln \left(u_{b}(t) v_{-}\right)=\ln v_{+}-\ln v_{-}=[M] .
$$

The equality $[L]=[M]$ implies that the $\lambda$-waves (32) are the same in coordinates $(c, L)$ and $(c, M)$. So, Theorem 6.1 is still valid replacing $L$ by $M$. We repeat the proof of Theorem 7.1 to get $B V$ estimates for $v$. 
8. Weak limit for velocity with $B V$ concentration. When $c$ is only in $B V$, we cannot reduce System (6) to a scalar conservation law for $c$ as in section 3. Indeed, since the shock speeds depend on the velocity, we have a true $2 \times 2$ hyperbolic system. Nevertheless we can state following stability result.

THEOREM 8.1 (Stability with respect to weak limit for the velocity). Let $\left(\ln \left(u_{b}^{\varepsilon}\right)\right)_{0<\varepsilon<1}$ be a bounded sequence in $L^{\infty}(0, T)$, such that

$$
u_{b}^{\varepsilon} \rightarrow \bar{u}_{b} \text { in } L^{\infty}(0, T) \text { weak }{ }^{*} \text {. }
$$

Let $c_{0} \in B V((0, X),[0,1])$ and $c_{b} \in B V((0, T),[0,1])$. Let $\left(c^{\varepsilon}, u^{\varepsilon}\right)$ be a weak entropy solution of System $(6)$ on $(0, T) \times(0, X)$ issuing from the FTA with initial and boundary values:

$$
\left\{\begin{array}{l}
c^{\varepsilon}(0, x)=c_{0}(x), \quad X>x>0 \\
c^{\varepsilon}(t, 0)=c_{b}(t), \quad T>t>0 \\
u^{\varepsilon}(t, 0)=u_{b}^{\varepsilon}(t), \quad T>t>0
\end{array}\right.
$$

Then, there exists $(u(t, x), c(t, x))$, a weak entropy solution of System (6), supplemented by initial and boundary values:

$$
\left\{\begin{array}{l}
c(0, x)=c_{0}(x), \quad x>0 \\
c(t, 0)=c_{b}(t), \quad t>0 \\
u(t, 0)=\bar{u}_{b}(t), \quad t>0
\end{array}\right.
$$

such that, when $\varepsilon$ goes to 0 , up to a subsequence:

$c^{\varepsilon}(t, x) \rightarrow c(t, x)$ strongly in $L^{1}([0, T] \times[0, X])$,

$u^{\varepsilon}(t, x) \rightarrow u(t, x)$ weakly in $L^{\infty}([0, T] \times[0, X])$ weak *,

$u^{\varepsilon}(t, x)=u_{b}^{\varepsilon}(t) \times v(t, x)+o(1)$ strongly in $L^{1}([0, T] \times[0, X])$, where $v(t, x)=\frac{u(t, x)}{\bar{u}_{b}(t)}$.

For the convergence of the whole sequence, we need the uniqueness of the entropy solution for the initial-boundary value problem: (6), (7).

Proof. From Theorem 7.2 we know that $u^{\varepsilon}(t, x)=u_{b}^{\varepsilon}(t) v^{\varepsilon}(t, x)$, where the sequences $\left(\ln v^{\varepsilon}\right)_{0<\varepsilon}$ and $\left(c^{\varepsilon}\right)_{0<\varepsilon}$ are uniformly bounded in $B V((0, T) \times(0, X))$. So, up to a subsequence, we have the following strong convergence in $L^{1}((0, T) \times(0, X)): v^{\varepsilon} \rightarrow v$, $c^{\varepsilon} \rightarrow c$.

$\left(c^{\varepsilon}, u^{\varepsilon}\right)$ is a weak entropy solution for (6) which means that for all $\psi$ such that $\psi " \geq 0$ and $Q$ such that $Q^{\prime}=h^{\prime} \psi+H \psi^{\prime}$ we have, in the distribution sense: $\partial_{x}\left(u^{\varepsilon}(t, x) \psi\left(c^{\varepsilon}\right)\right)+\partial_{t} Q\left(c^{\varepsilon}\right) \leq 0$, which is rewritten as follows: $\partial_{x}\left(u_{b}^{\varepsilon}(t) v^{\varepsilon}(t, x) \psi\left(c^{\varepsilon}\right)\right)+$ $\partial_{t} Q\left(c^{\varepsilon}\right) \leq 0$. Passing again to the weak-limit against a strong limit we get: $\partial_{x}\left(\bar{u}_{b}(t) v(t, x) \psi(c)\right)+\partial_{t} Q(c) \leq 0$. i.e. $\left(c, u=\bar{u}_{b} \times v\right)$ is a weak entropy solution for System (6). We can also pass to the limit on initial-boundary data.

Since there exists $\delta$ such that $0<\delta<u_{b}^{\varepsilon}<\delta^{-1}, v^{\varepsilon}(t, x) \rightarrow v(t, x)$ means that $u^{\varepsilon}(t, x) / u_{b}^{\varepsilon}(t)-v(t, x) \rightarrow 0$ and also that $u^{\varepsilon}(t, x)-u_{b}^{\varepsilon}(t) \times v(t, x) \rightarrow 0$, which concludes the proof. $\square$

An example of high oscillations for the velocity: As an example of weak limit, we consider the case of high oscillations for velocity on the boundary. 
Let $u_{b}(t, \theta) \in L^{\infty}\left((0, T), C^{0}(\mathbb{R} / \mathbb{Z}, \mathbb{R})\right), \bar{u}_{b}(t)=\int_{0}^{1} u_{b}(t, \theta) d \theta$ and assume that $\inf u_{b}>0$. With $u_{b}^{\varepsilon}(t)=u_{b}\left(t, \frac{t}{\varepsilon}\right)$ and the same notations as in Theorem 8.1 we have:

- first, oscillations do not affect the behavior of the concentration, since up to a subsequence, $\left(c^{\varepsilon}\right)$ converges strongly in $L^{1}$ towards $c$ and the limit system depends only on the average $\bar{u}_{b}$ and not on the oscillations, i.e. it is System (6) with initial boundary data (42);

- second, up to a subsequence, $\left(u^{\varepsilon}\right)$ converges weakly towards $\bar{u}_{b}(t) \times v(t, x)$ and we have a strong profile for $u^{\varepsilon}, U(t, x, \theta)=u_{b}(t, \theta) \times v(t, x)$ and

$$
\lim _{\varepsilon \rightarrow 0}\left\|u^{\varepsilon}(t, x)-U\left(t, x, \frac{t}{\varepsilon}\right)\right\|_{L^{1}((0, T) \times(0, X))}=0 .
$$

\section{REFERENCES}

[1] F. Ancona And A. Marson, Well-posedness for general $2 \times 2$ systems of conservation laws, Mem. Amer. Math. Soc., 169:801 (2004), pp. 1-170.

[2] F. AnCONA ANd A. Marson, Existence theory by front tracking for general nonlinear hyperbolic systems, Arch. Ration. Mech. Anal., 185:2 (2007), pp. 287-340.

[3] P. Bagnerini And M. Rascle, A multiclass homogenized hyperbolic model of traffic flow, SIAM J. Math. Anal., 35:3 (2003), pp. 949-973.

[4] P. Baiti and H. K. Jenssen, Well-posedness for a class of $2 \times 2$ conservation laws with $L^{\infty}$ data, J. Differential Equations, 140:1 (1997), pp. 161-185.

[5] S. Bianchini, Stability of $L^{\infty}$ solutions for hyperbolic systems with coinciding shock and rarefaction, SIAM J. Math. Anal., 33:4 (2001), pp. 959-981.

[6] C. Bourdarias, Sur un système d'edp modélisant un processus d'adsorption isotherme d'un mélange gazeux. (french) [on a system of p.d.e. modelling heatless adsorption of a gaseous mixture], M2AN, 26:7 (1992), pp. 867-892.

[7] C. Bourdarias, Approximation of the solution to a system modeling heatless adsorption of gases, SIAM J. Num. Anal., 35:1 (1998), pp. 13-30.

[8] C. Bourdarias, M. Gisclon, And S. Junca, A kinetic scheme for a $2 \times 2$ hyperbolic system arising in gas-solid chromatography, in preparation.

[9] C. Bourdarias, M. Gisclon, AND S. JunCA, Some mathematical results on a system of transport equations with an algebraic constraint describing fixed-bed adsorption of gases, J. Math. Anal. Appl., 313:2 (2006), pp. 551-571.

[10] C. Bourdarias, M. Gisclon, AND S. JunCA, Existence of weak entropy solutions for gas chromatography system with one or two actives species and non convex isotherms, Commun. Math. Sci., 5:1 (2007), pp. 67-84.

[11] C. Bourdarias, M. Gisclon, And S. JunCa, Hyperbolic models in gas-solid chromatography, Bol. Soc. Esp. Mat. Apl., 43 (2008), pp. 29-57.

[12] C. Bourdarias, M. Gisclon, And S. Junca, Blow up at the hyperbolic boundary for a $2 x 2$ chemical system, Journal of Hyperbolic Differential Equations (JHDE), 7:2 (2010), pp. 297316.

[13] A. Bressan, Global solutions of systems of conservation laws by wave-front tracking, J. Math. Anal. Appl., 170 (1992), pp. 414-432.

[14] A. Bressan, Hyperbolic Systems of Conservation Laws. The One-Dimensional Cauchy Problem, volume 20, Oxford University Press, 2008.

[15] G. Chen, S. Junca, And M. Rascle, Validity of nonlinear geometric optics for entropy solutions of multidimensional scalar conservation laws, J. Differential Equations, 222:2(2006), pp. $439-475$.

[16] C. Cheverry, O. Guès, And G. MÉTivier, Oscillations fortes sur un champ linéairement dégénéré (french), "strong oscillations on a linearly degenerate field", Ann. Sci. Ecole Norm. Sup., (4) 36:5 (2003), pp. 691-745.

[17] C. Cheverry, O. GuÈs, ANd G. MÉTIVIER, Large-amplitude high-frequency waves for quasilinear hyperbolic systems, Adv. Differential Equations, 9:7-8 (2004), pp. 829-890. 
[18] A. Corli And O. Guès, Local existence of stratified solutions to systems of balance laws, in Ann. Univ. Ferrara Sez., volume VII, pp. 109-119, Workshop on Partial Differential Equations, Ferrara, 1999.

[19] A. Corli And O. Guès, Stratified solutions for systems of conservation laws, Trans. Amer. Math. Soc., 353:6 (2001), pp. 2459-2486.

[20] C. Dafermos, Polygonal approximations of solutions of the initial value problem for a conservation law, J. Math. Anal. Appl., 38 (1972), pp. 33-41.

[21] C. Dafermos, Hyperbolic Conservation Laws in Continuum physics, Springer-Verlag, 2000.

[22] R. Diperna, Global existence of solutions to nonlinear hyperbolic systems of conservation laws, J. Differential Equations, 20 (1976), pp. 187-212.

[23] R. Diperna AND A. MAJDA, The validity of nonlinear geometric optics for weak solutions of conservation laws, Comm. Math. Phys., 98:3 (1985), pp. 313-347.

[24] O. Glass AND P.-G. LE FLOCH, Nonlinear hyperbolic systems: non-degenerate flux, inner speed variation and graph solutions, Arch. Ration. Mech. Anal., 185 (2007), pp. 409-480.

[25] J.-L. Joly, G. MÉtivier, AND J. RAUCH, Resonant one-dimensional nonlinear geometric optics, J. Funct. Anal., 114:1 (1993), pp. 106-231.

[26] S. JuncA, Réflexions d'ondes oscillantes semilinéaires monodimensionnelles, Comm. Partial Differential Equations, 23:3-4 (1998), pp. 727-759.

[27] S. JuncA, A two-scale convergence result for a nonlinear scalar conservation law in one space variable, Asymptot. Anal., 17:3 (1998), pp. 221-238.

[28] S. JuncA, Geometric optics with critical vanishing viscosity for one-dimensional semilinear initial value problems, Rev. Mat. Iberoamericana, 24:2 (2008), pp. 549-566.

[29] M. Douglas Levan, C. A. Costa, A. E. Rodrigues, A. Bossy, and D. Tondeur, Fixed-bed adsorption of gases: Effect of velocity variations on transition types, AIChE Journal, 34:6 (1988), pp. 996-1005.

[30] T. P. LiU, The entropy condition and the admissibility of shocks, J. Math. Anal. Appl., 53 (1976), pp. 78-88.

[31] A. Museux, Stratified weak solutions of the 1-d lagrangian euler equations are viscosity solutions, Adv. Differential Equations, 9:11-12 (2004), pp. 1395-1436.

[32] Y.-J. PENG, Explicit solutions for $2 \times 2$ linearly degenerate systems, Applied Mathematics Letters, 11:5 (1998), pp. 75-78.

[33] H. K. Rhee, R. Aris, And N.R. Amundson, On the theory of multicomponent chromatography, Philos. Trans. R. Soc. Lond., 267 (1970), pp. 419-455.

[34] H. K. Rhee, R. Aris, And N. R. Amundson, First-Order Partial Differential Equations, volume I, Prentice-Hall, 1986.

[35] N. H. Risebro, A front-tracking alternative to the random choice method, Proc. of the Amer. Math. Soc., 117 (1993), pp. 1225-1139.

[36] P. Rouchon, M. Sghoener, P. Valentin, and G. Guiochon, Numerical simulation of band propagation in nonlinear chromatography, in Chromatographic Science Series, volume 46, New York, 1988. Eli Grushka, Marcel Dekker Inc.

[37] P. M. Ruthwen, Principles of adsorption and adsorption processes, Wiley Interscience, 1984.

[38] D. Serre, Systèmes de lois de conservation I, Diderot, Paris, 1996.

[39] J. Smoller, Shock Waves and Reaction-Diffusion Equations, Springer Verlag, 1994.

[40] B. Temple, Systems of conservation laws with coinciding shock and rarefaction curves, in J. Smoller, editor, Nonlinear Partial Differential Equations, volume 17, pp. 143-151, Providence, RI, Amer. Math. Soc., 1983.

[41] B. Temple, Systems of conservation laws with invariant manifolds, Trans. Amer. Math. Soc., 280 (1983), pp. 781-795. 